\title{
靶向分枝菌酸生物合成的抗结核化合物研究进展
}

\author{
吕润秋张维 于丽芳* \\ (华东师范大学化学与分子工程学院 上海分子治疗与新药创制工程技术研究中心 上海 200062)
}

\begin{abstract}
摘要 结核病近年来已超过艾滋病成为当今世界范围内致死率高的感染性疾病之一. 结核病耐药问题严峻, 耐多药结 核病治愈率低、治疗方案复杂且周期长. 抗耐药结核领域存在重大研究机遇，函需发现与发展针对新靶点的新化学实 体. 分枝菌酸的生物合成和转运途径富含抗结核药物的有效靶点，综述了靶向该途径的抑制剂，讨论了其作用机制、药 代动力学性质等最新研究进展.
\end{abstract}

关键词 耐药结核病; 分枝菌酸的生物合成及转运途径; 重要靶点; 抑制剂

\section{Recent Advances in Antitubercular Compounds Targeting Mycolic Acid Biosynthesis and Transport}

\author{
Lü, Runqiu Zhang, Wei Yu, Lifang* \\ (Shanghai Engineering Research Center of Molecular Therapeutics and New Drug Development, School of Chemistry and \\ Molecular Engineering, East China Normal University, Shanghai 200062)
}

\begin{abstract}
Tuberculosis is now recognized as the most fatal infectious disease worldwide, eclipsing HIV in recent years. Drug-resistance tuberculosis continues to be a severe public crisis. The cure rate in persons with multidrug-resistant tuberculosis (MDR-TB) is unacceptably low, and the treatment of MDR-TB requires complex treatment regimens over a long period. There is a great opportunity in the field of tuberculosis as novel chemical entities with different mechanisms of action are particularly needed. The biosynthesis and transport pathways of mycolic acid represents a well proven and effective target for chemical intervention for TB. This article reviews the important inhibitors targeting to this pathway, as well as the recent research advances in the mechanism and pharmacokinetic properties etc.

Keywords drug-resistant tuberculosis; biosynthesis and transport pathway of mycolic acid; important targets; inhibitors
\end{abstract}

结核病 (Tuberculosis, TB) 是由结核分枝杆菌 (Mycobacterium tuberculosis, M. tb) 引起的感染性疾病, 结核病的致死率已经超过艾滋病, 是全球重大的公共卫 生问题之一. 据 WHO (World Health Organization)报道, 在 2019 年全球约有 1000 万例新发结核病, 其中非艾滋 病毒感染者中约有 120 万人死于结核病, 另有 20.8 万艾 滋病毒感染者死于结核病 ${ }^{11}$. 目前, 一线抗结核药物在 治疗药物敏感型结核病时的周期长, 患者依从性较差, 而二线抗结核药物在治疗以耐多药结核病(Multidrug resistant tuberculosis, MDR-TB)和广泛耐药结核病(Extensively drug resistant tuberculosis, XDR-TB)为代表的 耐药结核病患者时毒性大, 有效性也缺乏严格的证据 ${ }^{[2]}$. 因此, 开发新型抗结核药物来解决目前传统抗结核药物
治疗周期长, 对耐药结核病疗效差等问题具有重要的意 义.

$M . t b$ 为分枝杆菌属、放线菌门, 是一类形状细长略 微弯曲的杆菌 ${ }^{[3]} . M . t b$ 的细胞壁结构致密, 大多数抗菌 药物很难透过其细胞壁发挥抑菌效果 ${ }^{[4]} . M . t b$ 无内毒素, 也不产生外毒素和侵袭酶类 ${ }^{[5]}$, 其致病作用主要靠细胞 壁上大量的脂质复合物 ${ }^{[6]}$. 分枝菌酸(Mycolic acids, $\mathrm{MAs}$ )是构成 $M . t b$ 细胞壁上脂质复合物的重要组成部 分, 同时 MAs 与 $M . t b$ 在人体内的毒性和慢性感染有 关 ${ }^{[7]}$. 在抗结核药物研发中以分枝菌酸生物合成及转运 途径为靶点的优势在于此途径中的蛋白与人源蛋白差 异大, 对宿主细胞毒性低 ${ }^{[8]}$.

分枝菌酸是一种 $\alpha$-烷基- $\beta$-羟基疏水脂肪酸, 含有

* Corresponding author. E-mail: 1fyu@sat.ecnu.edu.cn

Received December 4, 2020; revised January 21, 2021; published online February 3, 2021.

Project supported by the National Natural Science Foundation of China (No. 21778019).

国家自然科学基金(No. 21778019)资助项目. 
一个碳数为 $22 \sim 26$ 的短臂和一个碳数为 $50 \sim 60$ 的长 臂 ${ }^{[9]}$. 在结核杆菌细胞壁上 MAs 与阿拉伯半乳聚糖通过 酯键相连, 同时 MAs 与外膜上的其它糖类结合为海藻 糖单分枝菌酸酯(Trehalose monomycolate, TMM)、海藻 糖二分枝菌酸酯(Trehalose dimycolate, TDM)和葡萄糖 单分枝菌酸酯(Glucose monomycolate, GMM)等 ${ }^{[10]}$. 根
据分枝菌酸结构中带有基团的不同一般分为 3 个类型: $\alpha$-分枝菌酸(1)、甲氧基分枝菌酸 $(2,3)$ 和酮式分枝菌酸 $(4$, 5) ${ }^{[11]}$ (Scheme 1).

分枝菌酸的生物合成过程发生在细胞质中(图 1). I 型脂肪酸合酶(Fatty acid synthase-I, FAS-I)合成 2 条酰 基-辅酶 $\mathrm{A}(\mathrm{CoA})$ 脂肪酸链，一条形成链长 $\mathrm{C} 24 \sim \mathrm{C} 26$ 的

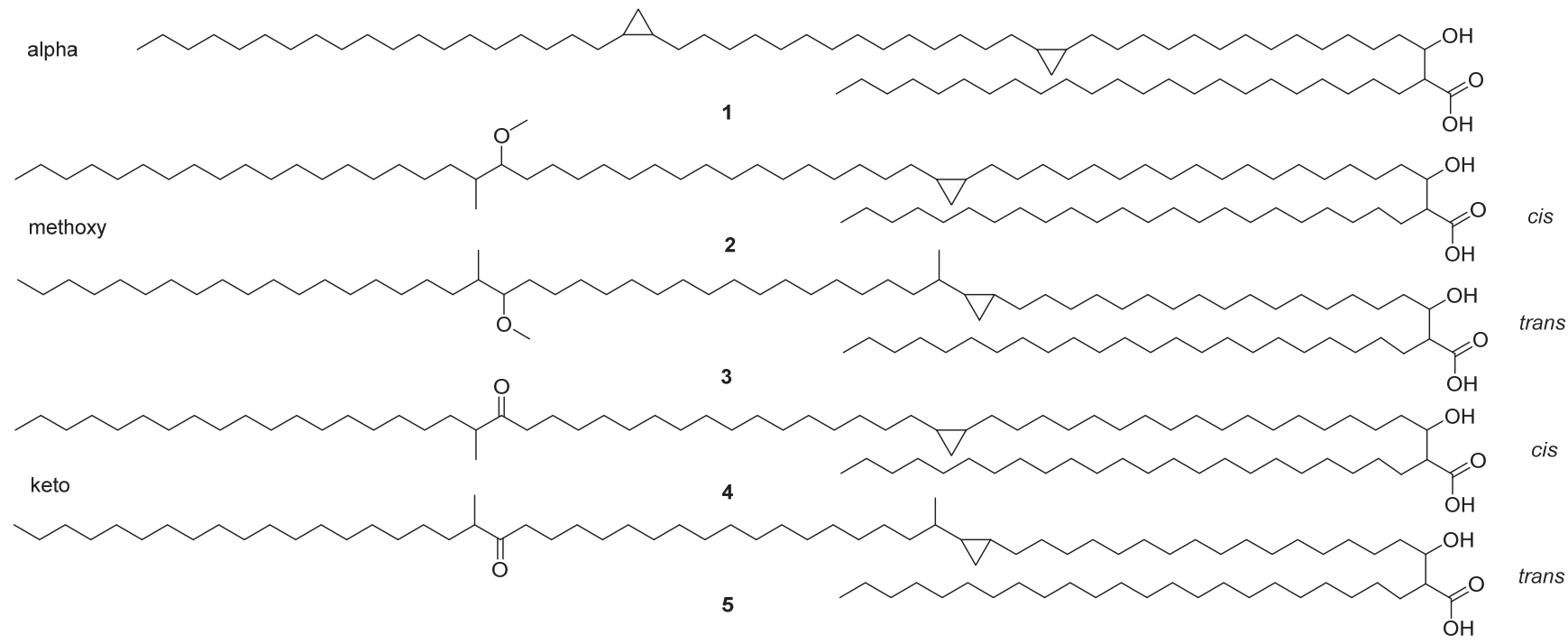

图式 1 分枝菌酸的结构

Scheme 1 Structures of mycolic acids

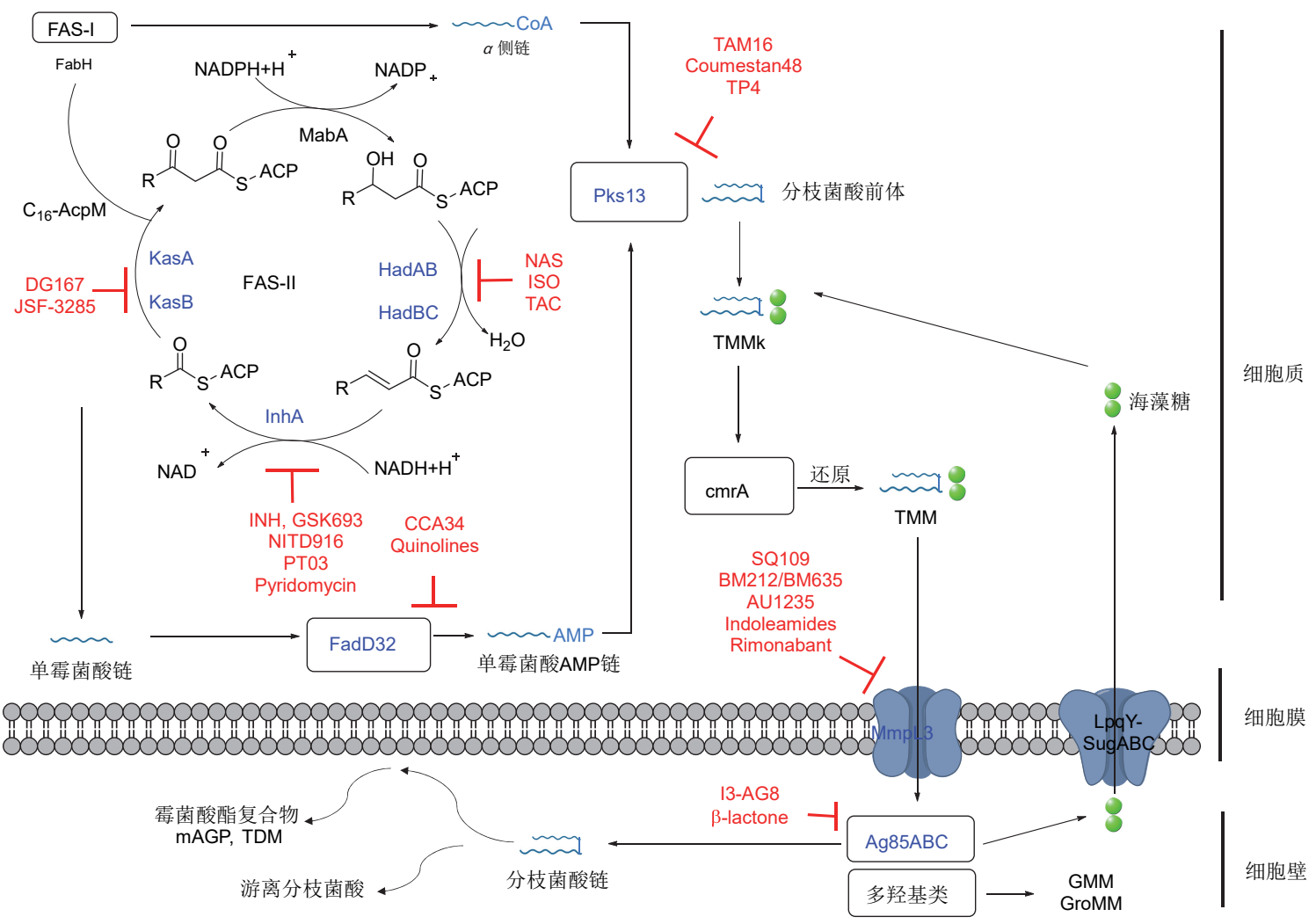

图 1 分枝菌酸的合成和转运途径以及相关抗结核靶点和抑制剂

Figure 1 Synthesis and transport pathways of mycolic acid and related antituberculosis targets and inhibitors 
饱和 $\alpha$-支链, 另一条链通过 $\beta$-酮脂酰基载体蛋白合酶 (FabH)和丙二酰一分枝杆菌酰基载体蛋白链 (AcpM) 缩合 产生 C16-AcpM 链 ${ }^{[2]} . M$. $t b$ 中的 II 型脂肪酸合酶(Fatty acid synthase-II, FAS-II)主要由四种酶组成, $\beta$-酮酯酰基 ACP 合成酶(KasA、KasB, $R v 1745 、 R v 2246)^{[13]}$, 酮还原 酶 (MabA，Rv1483 $)^{[14] ，} \beta$ - 羟烷基脱水酶 (HadABC, $R v 0635 、 R v 0636 、 R v 0637)$ 和烯酰 ACP 还原酶( InhA, $R v 1484)^{[15]}$ 等. 其中 MabA、HadABC 和 InhA 经过一系 列还原、脱水等步骤将 C16-AcpM 的饱和脂肪链与 AcpM 连接生成 C18-AcpM, KasA 和 KasB 负责将 C18-AcpM 运送至 FAS-II 的起点进行循环, 每个循环过 程在 AcpM 上增加 2 个碳原子最终生成 C50 C60-AcpM ${ }^{[16-17]}$. C $50 \sim \mathrm{C} 60-\mathrm{AcpM}$ 由脂肪酸降解蛋白 (FadD32)作用后形成分枝菌酸-AMP 链 ${ }^{[18]}$. 聚酮合酶 13(Pks13)催化分枝菌酸-AMP ( $\beta$-支链)与酰基-CoA(饱 和 $\alpha$-支链)发生克莱森缩合反应, 生成分枝菌酸前体 ${ }^{[19]}$.

Pks13 硫酯酶(thioesterase, TE)结构域运送分枝菌酸 前体到海藻糖上形成酮式海藻糖分枝菌酸酯(Trehalose ketomonomycolate, TMMk), TMMk 由还原酶(CmrA)还 原形成 $\mathrm{TMM}^{[20]}$, 接着 $\mathrm{TMM}$ 被膜转运蛋白 $(\mathrm{MmpL} 3)$ 转 运到细胞膜外 ${ }^{[21]}$. 抗原 $85(\mathrm{Ag} 85)$ 在膜外将 TMM 上的分 枝菌酸链转移细胞膜上形成重要的分枝菌酸酯类化合 物 ${ }^{[22]}$, 如: 分枝菌酸酯-阿拉伯糖-肽聚糖复合物 (mycolyl-arabinogalactan-peptidoglycan, mAGP)、TDM、 GMM 和甘油单分枝菌酸酯(Glycerin monomycolate, GroMM) 等; 同时游离的海藻糖 经 细胞膜上的 LpqY-SugA-SugB-SugC ABC 转运蛋白输送回细胞质, 形成循环过程 ${ }^{[23]}$.

临床上使用的抗结核药物很多是靶向分枝菌酸生 物合成途径的, 包括异烟肼(isoniazid, INH)、乙硫异烟胺 (ethionamide)和德拉马尼(delamanid)等. 异烟肼自 1952 年问世后便作为结核病治疗的一线药物. 它是一种前 药, 需要被 KatG 激活产生自由基再与烟酰胺腺嘌呤二 核苷酸(NAD)形成共价加合物来抑制 InhA. 由于 KatG 中的 Ser315 易发生突变, 导致 INH 在使用过程中容易 出现耐药 ${ }^{[24]}$. 乙硫异烟胺是一种 INH 的类似物, 需要经 EthA (FAD 依赖性单加氧酶)活化后作用于 InhA ${ }^{[25]}$. 德 拉马尼于 2014 年被欧盟批准上市用于 MDR-TB 的治疗, 它需要通过细胞内的异菌脱氮腿依赖的硝基还原酶进 行生物活化，进而抑制分枝菌酸的生物合成. ${ }^{[26]}$

\section{1 重要靶点及其抑制剂}

\subsection{KasA 及其抑制剂}

KasA 属于 $\beta$-酮酯酰基合酶, 由 435 个氨基酸组成, 它负责延长酰基 ACP 的碳链. KasA 的晶体结构于 2016
年被解析出来(PDB ID: 5LD8). Kumar 等 ${ }^{[27]}$ 在 2018 年报 道了苯并吡唑磺胺类分子 DG167 (6) 靶向抑制 KasA, 6 在 KasA 的结合口袋中以双分子形式占据不同位点，阻 止酶与底物结合 ${ }^{[28]} .6$ 对野生型 H37Rv 菌株的 MIC 值为 $0.39 \mu \mathrm{mol} / \mathrm{L}$, 同时与一线抗结核药物无交叉耐药. 然而 在动物实验中口服给药并不能明显降低 BALB/c 小鼠肺 部细菌的数量, 利用液相-质谱联用监测发现 6 芳环上 的甲基在体内易代谢脱去，进而导致体内药效不佳. 于 是 6 的结构被优化为 JSF-3285 (7, $\mathrm{MIC}_{\mathrm{H} 37 \mathrm{Rv}}$ : 0.20 $\mu \mathrm{mol} / \mathrm{L}$ ), 在雌性 CD-1 小鼠感染 H37Rv 菌株四周后以 5 $\mathrm{mg} / \mathrm{kg}$ 的剂量口服给药 7, 与空白对照组相比能够使肺 部菌落数量减少, $\mathrm{AUC}_{0 \sim 5 \mathrm{~h}}$ 大幅提升至 $59323(\mathrm{ng} / \mathrm{mL}) \cdot \mathrm{h}$, 小鼠肝微粒体代谢半衰期由 $10.1 \mathrm{~min}$ 增加至 $28.4 \mathrm{~min}^{[29]}$ (Scheme 2). 此外, 在小鼠模型中优选化合物 7 的浓度 大于 MIC 的时间为 $5 \mathrm{~h}$; 在 $5 \mathrm{~h}$ 时肺部药物浓度与血浆 药物浓度的比值为 0.75 . 在毒性上, 7 对 Vero 细胞的 $\mathrm{CC}_{50}$ 值为 $170 \mu \mathrm{mol} / \mathrm{L}$, 对 $\mathrm{hERG}$ 钾通道的 $\mathrm{IC}_{50}$ 值大于 50 $\mu \mathrm{mol} / \mathrm{L}$ (表 1).
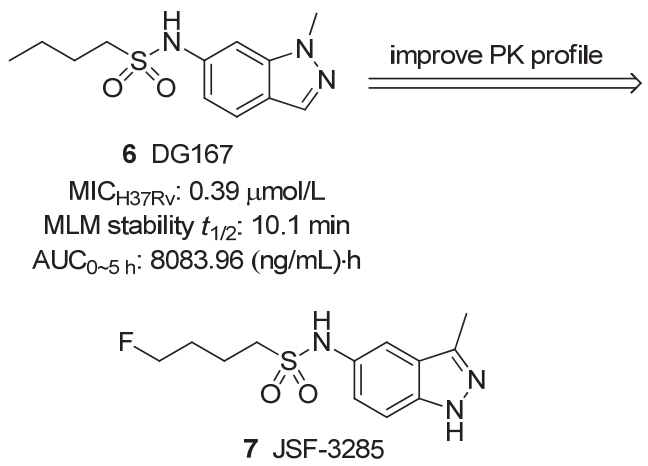

$$
\begin{gathered}
\text { MIC }_{\text {H37Rv: }}: 0.20 \mu \mathrm{mol} / \mathrm{L} \\
\text { MLM stability } t_{1 / 2}: 28.4 \mathrm{~min}
\end{gathered}
$$
$\mathrm{AUC}_{0 \sim 5} \mathrm{~h}: 59323(\mathrm{ng} / \mathrm{mL}) \cdot \mathrm{h}$

图式 2 DG167 和 JSF-3285

Scheme 2 DG167 and JSF-3285

表 1 已报道的吸收、分布、代谢、排泄、毒性(AbsorptionDistribution-Metabolism-Excretion-Excretion, ADMET) Table 1 Reported Absorption-Distribution-Metabolism-Excretion-Excretion properties of JSF-3285

\begin{tabular}{ll}
\hline Vero $\mathrm{CC}_{50}$ & $170 \mu \mathrm{mol} / \mathrm{L}$ \\
$\mathrm{hERG} \mathrm{IC}_{50}$ & $>50 \mu \mathrm{mol} / \mathrm{L}$ \\
$t($ Cplasma $>\mathrm{MIC})$ & $5 \mathrm{~h}$ \\
$C_{\text {lung }} / C_{\text {plasma }}$ at $5 \mathrm{~h}$ & 0.75 \\
\hline
\end{tabular}

\section{$1.2 \operatorname{Inh} A$ 及其抑制剂}

烯酰 ACP 还原酶(InhA)属于短链脱氢酶家族, 由 268 个氨基酸组成, 其晶体结构于 1998 年被报道(PDB ID: $1 \mathrm{BVR})^{[30]}$. InhA 可以由烟酰胺腺嘌呤二核苷酸还原 型辅酶 I 提供能量将脂肪酰基底物上 $\mathrm{C}(2)$ 和 $\mathrm{C} 3$ 间的反 式双键还原 ${ }^{[31]}$, 而 InhA 的失活会导致二十四烷酸堆积 
以及分枝杆菌细胞壁结构变化. 一线抗结核药物 INH 产 生耐药性与活化酶 KatG 的 Ser315 突变有关, 所以目前 许多研究小组致力于开发直接靶向 InhA 的抑制剂. 然 而大多数化合物的酶抑制活性与细胞水平活性不相关, 选择性低、药代动力学性质差, 使得它们不适合进行更 深入的研究 ${ }^{[32]}$. GSK 公司篎选得到的噻二唑类化合物 GSK613 (8) 对 InhA 酶和 H37Rv 菌株均表现出较强的抑 制活性 $\left(\mathrm{IC}_{50}: 7 \mathrm{nmol} / \mathrm{L}, \mathrm{MIC}_{\mathrm{H} 37 \mathrm{Rv}} \leqslant 1 \mu \mathrm{mol} / \mathrm{L}\right)$, 该类化合 物属于竞争性抑制剂[33]. 由于 8 的水溶性差、血药浓度 低(Scheme 3), GSK 公司对 8 进行结构改造得到 GSK693 $\left(9, \mathrm{IC}_{50}: 7 \mathrm{nmo} / \mathrm{L}, \mathrm{MIC}_{\mathrm{H} 37 \mathrm{Rv}}: 0.2 \mu \mathrm{mol} / \mathrm{L}\right), 9$ 在 $\mathrm{C} 57 \mathrm{BL} / 6 \mathrm{j}$ 小鼠体内最大药物浓度提高至 $37271 \mathrm{ng} / \mathrm{mL}$, 小鼠肝微 粒体清除率降低至 $2.1 \mathrm{~mL} \cdot \mathrm{min}^{-1} \cdot \mathrm{g}^{-1}$ (Scheme 4). 在毒 性上, 优选化合物 9 对 CYP450 3A4 亚型的 $\mathrm{IC}_{50}$ 值大于 $50.1 \mu \mathrm{mol} / \mathrm{L}$, 对 $\mathrm{HepG} 2$ 细胞和心血管的 $\mathrm{IC}_{50}$ 值都大于 $50 \mu \mathrm{mol} / \mathrm{L}$. 在小鼠模型中以 $4 \mathrm{mg} / \mathrm{kg}$ 的剂量静脉注射 $\mathbf{9}$, 它在血浆稳态下的表观分布体积为 $2.58 \mathrm{~L} / \mathrm{kg}$, 半衰期为 $0.94 \mathrm{~h}, 9$ 的其它 ADMET 参数见表 2 .

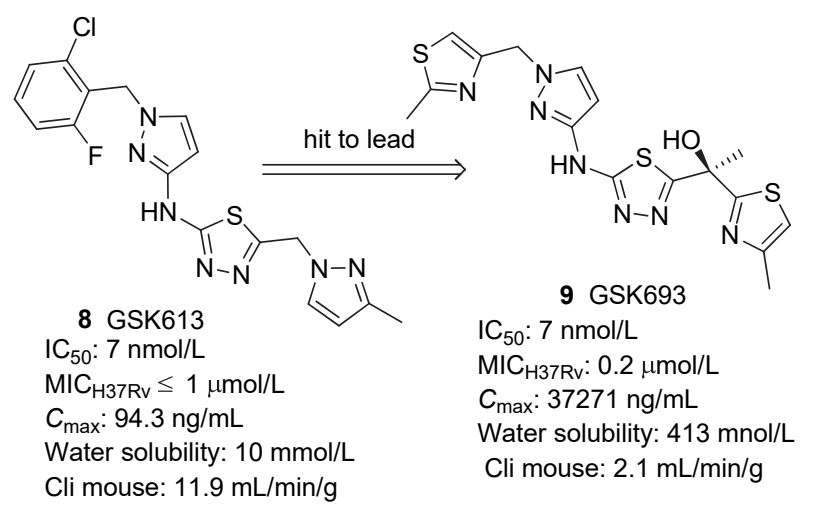

图式 3 噻二唑类化合物的结构

Scheme 3 Structures of thiadiazoles

表 2 GSK693 的已报道 ADMET 性质

Table 2 Reported ADMET properties of GSK693

\begin{tabular}{|c|c|}
\hline CYP450 3A4 IC50 & $>50.1 \mu \mathrm{mol} / \mathrm{L}$ \\
\hline HepG2 Cytotoxicity $\mathrm{IC}_{50}$ & $>50 \mu \mathrm{mol} / \mathrm{L}$ \\
\hline Ames test & Negative \\
\hline Cardiovascular profile Qpatch $\mathrm{IC}_{50}$ & $>50 \mu \mathrm{mol} / \mathrm{L}$ \\
\hline Cli human (in vitro) & $0.2 \mathrm{~mL} \cdot \mathrm{min}^{-1} \cdot \mathrm{g}^{-1}$ \\
\hline Vss $(4 \mathrm{mg} / \mathrm{kg} \text { iv })^{a}$ & $2.58 \mathrm{~L} / \mathrm{kg}$ \\
\hline$t_{1 / 2}(4 \mathrm{mg} / \mathrm{kg}$ iv $)$ & $0.94 \mathrm{~h}$ \\
\hline$T_{\max }(100 \mathrm{mg} / \mathrm{kg}$ po $)$ & $0.42 \mathrm{~h}$ \\
\hline DNAUC $(\text { in vivo })^{b}$ & $935.8(\mathrm{ng} \bullet \mathrm{h}) / \mathrm{mL}$ per $\mathrm{mg} / \mathrm{kg}$ \\
\hline
\end{tabular}

天然产物吡啶霉素(10)于 2012 年也被证明对 InhA 有抑制作用 $\left(\mathrm{IC}_{50}: 18.6 \mu \mathrm{mol} / \mathrm{L}, \mathrm{MIC}_{\mathrm{H} 37 \mathrm{Rv}}: 0.39 \mu \mathrm{g} / \mathrm{mL}\right)$, 并且 10 对人源细胞毒性较小 ${ }^{[34]}$. 此外, 还有 4-差基-2吡啶酮类 $\left[11, \mathrm{IC}_{50}:(0.57 \pm 0.04) \mu \mathrm{mol} / \mathrm{L}, \mathrm{MIC}_{99, \mathrm{H} 37 \mathrm{Rv}}: 0.08\right.$ $\mu \mathrm{mol} / \mathrm{L}]$ ，二芳基醚类 $\left[12 ， \mathrm{IC}_{50} ： 5 \mathrm{nmol} / \mathrm{L}, \mathrm{MIC}_{\mathrm{H} 37 \mathrm{Rv}}\right.$ ：1.0 $\mu \mathrm{g} / \mathrm{mL}]$ 等被报道靶向抑制 $\operatorname{Inh}^{[35-36]}$ (Scheme 4).

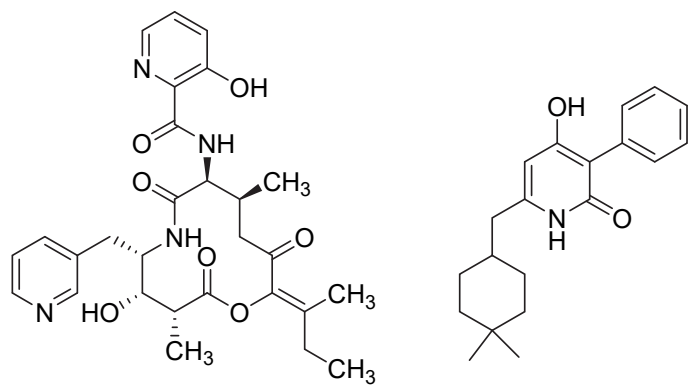

10 pyridomycin

11 NITD916

$I_{50}: 18.6 \mu \mathrm{mol} / \mathrm{L} \quad \mathrm{IC}_{50}: 0.57 \pm 0.04 \mu \mathrm{mol} / \mathrm{L}$ $\mathrm{MIC}_{\mathrm{H} 37 \mathrm{Rv}}: 0.39 \mu \mathrm{g} / \mathrm{mL} \quad \mathrm{OH} \quad \mathrm{MIC}_{99, \mathrm{H} 37 \mathrm{Rv}}: 0.08 \mu \mathrm{mol} / \mathrm{L}$<smiles>CCCCCc1ccc(Oc2ccccc2)c(O)c1</smiles>

12 PT03

$\mathrm{IC}_{50}: 5 \mathrm{nmol} / \mathrm{L}$

$\mathrm{MIC}_{\mathrm{H} 37 \mathrm{Rv}}: 1.0 \mu \mathrm{g} / \mathrm{mL}$

图式 4 其它靶向 InhA 的化合物

Scheme 4 Other compounds target to InhA

\section{$1.3 \mathrm{HadABC}$ 及其抑制剂}

异源二聚体 $\mathrm{HadAB}$ 和 $\mathrm{HadBC}$ 分别由 287 和 300 个 氨基酸组成 ${ }^{[37]}$, 在脂肪酸延伸循环过程中 $\operatorname{HadAB}$ 负责 催化形成 $\mathrm{C}(22) \sim \mathrm{C}(42)$ 的分枝菌酸链, HadBC 负责延长 碳链个数至 $\mathrm{C}(52) \sim \mathrm{C}(64)$, 它们的晶体结构分别于 2015 年(PDB ID: 4RV2)和 2018 年(PDB ID: 5ZY8)被解析出 来. Bhowruth 等 ${ }^{[38]}$ 报道 NAS-21 (13)类化合物具有抗结 核活性. NAS-21 衍生物中双羰基侧链为活性必需, 将其 替换为乙酰基时活性消失，推测可能有两种原因: (1)双 羰基结构衍生物与 $\beta$ 酮底物相似，在 $\operatorname{HadABC}$ 参与的生 物途径中作为竞争性抑制剂; (2) $\mathrm{CF}_{3}$ 基团能使酮一烯醇 互变异构体稳定，从而与活性位点中的氨基酸残基产生 相互作用. 在体外的 FAS-II 抑制试验中 13 的类似物 $\mathrm{IC}_{50}$ 值达到了 $19 \mu \mathrm{g} / \mathrm{mL}$. 该类化合物通过抑制 $\operatorname{HadABC}$ 在 生物合成中的脱水作用，从而影响分枝菌酸的合成. 此 外, 戊氧苯硫脲 $\left(14, \mathrm{ISO}, \mathrm{MIC}_{\mathrm{H} 37 \mathrm{Rv}}: 2.5 \mu \mathrm{mol} / \mathrm{L}\right)$ 和氨硫嫝 $\left(\mathbf{1 5}, \mathrm{TAC}, \mathrm{MIC}_{\mathrm{H} 37 \mathrm{Rv}}: 0.5 \mu \mathrm{mol} / \mathrm{L}\right)$ 这两个前药由 EthA 活化 靶向抑制 $\operatorname{HadABC}$, 然而由于 14 在体内吸收差、生物利 用度低, 15 的毒性大而未能走得更远 ${ }^{[39]}$ (Scheme 5).

\subsection{FadD32 及其抑制剂}

脂肪酰基-AMP 连接酶 FadD32 由含 492 个氨基酸 的 $\mathrm{N}$ 末端结构域和含 144 个氨基酸的 $\mathrm{C}$ 末端结构域组 成，负责活化由 FAS-II 合成的分枝菌酸长链，随后参与 Pks13 介导的克莱森缩合反应 ${ }^{[40]}$. Kuhn 等 ${ }^{[41]}$ 利用 PhU-AMS 配体(16) (Scheme 6) 解析出 FadD32 的晶体结 构(PDB ID: 5HM3). Stanley 等[42]在 2013 年通过全细胞 
<smiles>O=C(CC(=O)C(F)(F)F)c1ccc([N+](=O)[O-])cc1</smiles>

$\mathrm{MIC}_{99, \mathrm{H} 37 \mathrm{Rv}}: 63 \mu \mathrm{g} / \mathrm{mL} \mathrm{IC}{ }_{50}: 94 \mu \mathrm{g} / \mathrm{mL}$

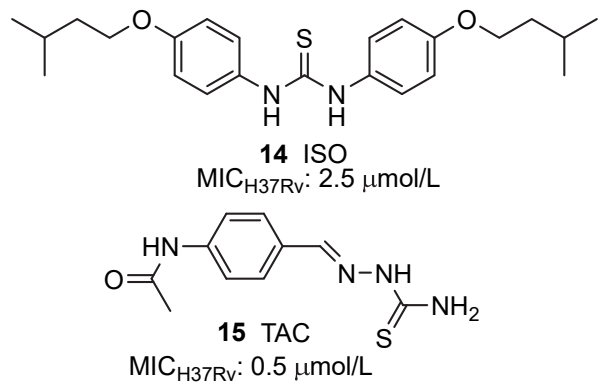

图式 $5 \mathrm{HadABC}$ 抑制剂

Scheme 5 HadABC inhibitors
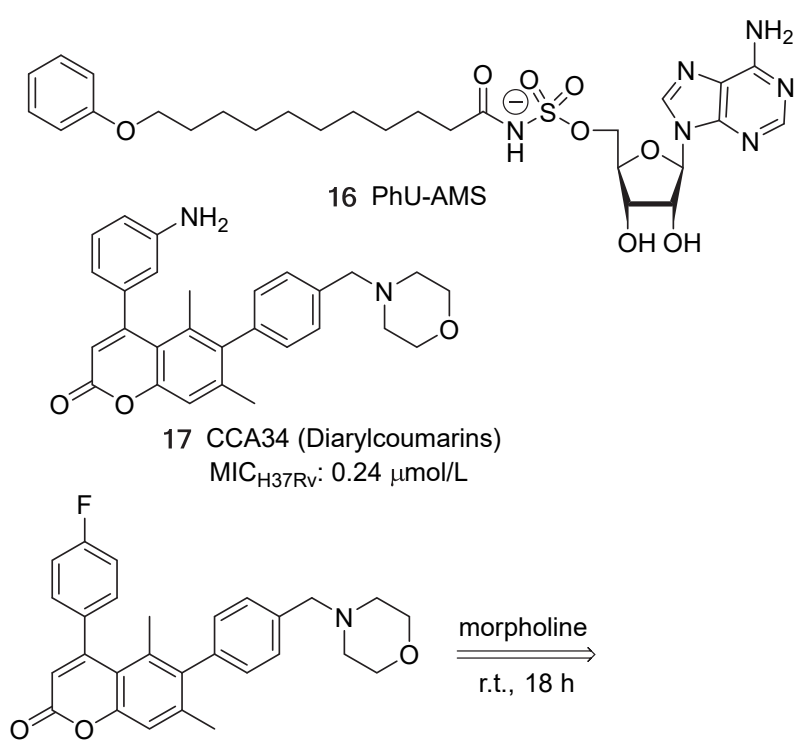

18<smiles>Cc1cc(O)c(/C(=C\C(=O)N2CCOCC2)c2ccc(F)cc2)c(C)c1-c1ccc(CN2CCOCC2)cc1</smiles><smiles>CNC(=O)c1cc(-c2ccccc2Cl)c2c(C)c(-c3ccncc3)c(C)cc2n1</smiles>

图式 6 PhU-AMS (16), CCA34 (17)和喹啉-2-酰胺(20)的结构 以及香豆素母核 $(18)$ 在碱性条件下开环

Scheme 6 Structures of PhU-AMS (16), CCA34 (17), and quinoline-2-carboxamides (20), and coumarin core (18) underwent ring opening under basic condition
篮选发现二芳基香豆素类衍生物是一类有效的 FadD32 抑制剂 $\left(\mathrm{IC}_{90}\right.$ : $\left.36 \mu \mathrm{mol} / \mathrm{L}\right)$, 其中化合物 $\mathrm{CCA} 34$ (17)对 $\mathrm{H} 37 \mathrm{Rv}$ 菌株的 MIC 值达到 $0.24 \mu \mathrm{mol} / \mathrm{L}$ (Scheme 6), 在 $\mathrm{BALB} / \mathrm{c}$ 小鼠感染 Erdman 菌株 $7 \mathrm{~d}$ 后以 $35 \mathrm{mg} / \mathrm{kg}$ 的剂 量腹腔注射 17 能使肺部菌落数量减少 1 个 $\log$ CFU.

Fang 等[43]考虑到香豆素母核上的内酯容易在体内 形成共价加合物引起毒性，并且当香豆素母核上存在吸 电子基团时, 18 在碱性条件下不稳定容易开环成为 19 (Scheme 6). 于是他们将香豆素母核替换为喹啉环, 结 构稳定性得到改善, 并且在喹啉环上更容易进行取代基 的拓展和优化. 经改造得到的喹啉-2- 甲酰胺 (20, $\mathrm{MIC}_{\mathrm{H} 37 \mathrm{Rv}}$ : $0.8 \mu \mathrm{mol} / \mathrm{L}$ )对 $\mathrm{H} 37 \mathrm{Rv}$ 菌株的抑制活性与 17 基 本持平, 并且对人源细胞的毒性较小 $\left(\mathrm{HepG} 2 \mathrm{IC}_{50}\right.$ : 60 $\mu \mathrm{mol} / \mathrm{L}$ ), 有望进行更深入的研究. 此外, Galandrin 等 ${ }^{[4]}$ 利用化合物对酶热稳定性的影响篮选得到五类结构对 FadD32 有抑制作用, 分别为苯并咪唑类、硫代嘧啶类、 吡咯唑类、喹诺酮类和异喹诺酮类.

\subsection{Pks13 及其抑制剂}

聚酮合酶 Pks13 包含 1733 个氨基酸, 分子质量为 $186 \mathrm{kDa}$. Pks13 由 5 个结构域组成, 分别为酩酰基合成 酶(KS)域、乙酰转移酶(AT)域、C 端酰基载体蛋白域 (C-ACP)、 $\mathrm{N}$ 端酰基载体蛋白域(N-ACP)和硫酷酶(TE) 结构域 ${ }^{[45]}$. Pks13 是催化分枝菌酸生物合成最后一步的 关键酶，是抗结核药物研发中发现的一个新靶点.

2013 年 Wilson 等 ${ }^{[46]}$ 报道了第一个具有抗结核活性 的噻吩类 Pks13 抑制剂，其中代表性化合物 TP2(21)对 $\mathrm{H} 37 \mathrm{Rv}$ 菌株的 MIC 为 $1.0 \mu \mathrm{mol} / \mathrm{L}$. 抗性突变体进行全基 因组测序发现突变位点 F79S 位于 Pks13 的 N-ACP 区域 附近，推测此类化合物是通过抑制 Pks13 中 N-ACP 上 P-pant 臂装载功能影响了 FadD32 上的分枝菌酸-AMP 链的装载. Thanna 等 ${ }^{[47]}$ 发现将环庚基替换为含氮杂环后 $\mathrm{MIC}_{\mathrm{H} 37 \mathrm{Rv}}$ 降至 $0.69 \mu \mathrm{mol} / \mathrm{L}$, 并且 22 在 THP-1 细胞内 $\left(\mathrm{IC}_{90} ： 9.75 \mu \mathrm{mol} / \mathrm{L}\right)$ 和低氧条件下 $\left(\mathrm{IC}_{90}: 1.5 \mu \mathrm{mol} / \mathrm{L}\right)$ 对 H37Rv 菌株都有较好的抑制作用(Scheme 7).

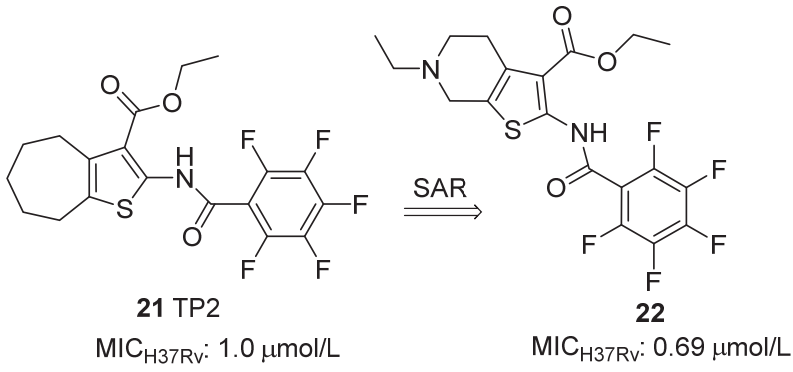

图式 7 噻吩类 Pks13 抑制剂

Scheme 7 Thiophene series of Pks13 inhibitors

2013 年 Ioerger 等 ${ }^{[48]}$ 报道了苯并呋喃类化合物 
TAM1 [23, IC $\mathrm{IC}_{50}:(0.26 \pm 0.03) \mu \mathrm{mol} / \mathrm{L}, \mathrm{MIC}_{\mathrm{H} 37 \mathrm{Rv}}: 2.3$ $\mu \mathrm{mol} / \mathrm{L}]$ 靶向抑制 Pks13, 对抗性突变体进行全基因测序 发现突变位点位于 Pks13 的 TE 域. Sacchettini 课题组 ${ }^{[49]}$ 利用生物化学方法验证了苯并呋喃类化合物对 Pks13TE 具有抑制作用, 解析了化合物与 Pks13-TE 的共晶复 合物，设计合成的 TAM16 (24)对 H37Rv 菌株有很强的 抑制活性(MIC: $0.09 \mu \mathrm{mol} / \mathrm{L})$, 对耐药菌株的抑制也同 样高效. 在小鼠急、慢性感染模型中, 24 分别在 200 和 $300 \mathrm{mg} / \mathrm{kg}$ 的口服剂量下可显著降低肺部的结核杆菌菌 落数量. 24 对细胞色素 P450酶各亚型无明显抑制, 利用 $\mathrm{TI}+$ 通量测定法发现其对 hERG 钾通道的影响不大 $\left(\mathrm{IC}_{50}: 21 \mu \mathrm{mol} / \mathrm{L}\right)$, 同时在小鼠模型中连续 $3 \mathrm{~d}$ 单次给药 $300 \mathrm{mg} / \mathrm{kg}$ 以下无明显急性毒性 ${ }^{[49]}$. 优选化合物 24 在小 鼠模型中, 以 $10 \mathrm{mg} / \mathrm{kg}$ 的剂量口服给药, 24 在血浆中的 最大浓度为 $444 \mathrm{ng} / \mathrm{mL}$, 药物达到最高浓度的时间为 0.5 $\mathrm{h}$, 小鼠的 $\mathrm{AUC}_{0 \sim 24}$ 值为 $75 \mu \mathrm{g} \cdot \mathrm{min} / \mathrm{mL}$, 口服生物利用 度达到 $28 \%$ (表 3$)^{[49]}$.

表 3 TAM16 的已报道 ADMET 性质 Table 3 Reported ADMET properties of TAM16

\begin{tabular}{ll}
\hline Plasma protein binding & Mouse $73 \%$ \\
& Human $72 \%$ \\
CYP inhibition & No significant inhibition \\
$c_{\max }$ in plasma $(10 \mathrm{mg} / \mathrm{kg} \mathrm{po})$ & $444 \mathrm{ng} / \mathrm{mL}$ \\
$T_{\max }(10 \mathrm{mg} / \mathrm{kg} \mathrm{po})$ & $0.5 \mathrm{~h}$ \\
$\mathrm{AUC}_{0-24}(10 \mathrm{mg} / \mathrm{kg} \mathrm{po})$ & $74940 \mathrm{ng} \cdot \mathrm{min} / \mathrm{mL}$ \\
$\mathrm{AUC}_{0-24}(3 \mathrm{mg} / \mathrm{kg}$ iv) & $79369 \mathrm{ng} \bullet \mathrm{min} / \mathrm{mL}$ \\
${\text { Vss }(3 \mathrm{mg} / \mathrm{kg} \mathrm{iv})^{a}}$ & $4.2 \mathrm{~L} / \mathrm{kg}$ \\
Oral bioavailability $(F)$ & $28 \%$ \\
\hline
\end{tabular}

${ }^{a}$ Volume of distribution at steady state.

我们课题组发现苯并呋喃衍生物 3 位为乙酯时, 化 合物在小鼠体内容易水解失活. 我们将天然产物 coumestan 骨架合理应用于 Pks13 抑制剂的设计合成, 发展 了体内有效的类天然产物 coumestan 抗结核活性化合 物 ${ }^{[50]}$ (Scheme 8), 其中 coumestan48 (25)的 $\mathrm{MIC}_{\mathrm{H} 37 \mathrm{Rv}}$ 值 达到 $3.9 \mathrm{ng} / \mathrm{mL}$, 同时 $\mathbf{2 5}$ 的半衰期和人源肝微粒体代谢 稳定性大幅提升. 在小鼠血清滴定抑制实验中以 100 $\mathrm{mg} / \mathrm{kg}$ 的剂量口服给药, 25 的活性优于 $\mathbf{2 4}$, 并且 25 对其 它代表性致病菌(金黄色葡萄球菌、大肠杆菌、枯草芽 孢杆菌)有较好的选择性. 此外, 25 对药物敏感性菌株 (V4207 MIC: $0.0078 \mu \mathrm{mol} / \mathrm{L}) 、 广$ 广泛耐药菌株(TF274 MIC: $0.0078 \mu \mathrm{mol} / \mathrm{L}, \mathrm{R} 506 \mathrm{MIC}: 0.0078 \mu \mathrm{mol} / \mathrm{L})$ 和耐多 药菌株 (KZN494 MIC: $0.0039 \mu \mathrm{mol} / \mathrm{L}, \mathrm{V} 2475 \mathrm{MIC}$ : $0.0156 \mu \mathrm{mol} / \mathrm{L})$ 的抑制效果同样高效 ${ }^{[51]}$. 在毒性上, 25 对 Vero 细胞的 $\mathrm{IC}_{50}$ 值为 $4 \mu \mathrm{g} / \mathrm{mL}$, 对人源细胞株 MRC-5、 HFL-1、HEK-293 的 $\mathrm{IC}_{50}$ 值都是大于 $50 \mu \mathrm{g} / \mathrm{mL}$ ，对人源 细胞株 QSG-7701 的 $\mathrm{IC}_{50}$ 值为 $(40.1 \pm 3.5) \mu \mathrm{g} / \mathrm{mL}^{[51]} .2019$ 年 Molin 等[52]通过表型笁选得到吲哚骨架 26 对 H37Rv 菌株的 MIC 值达到 $0.244 \mu \mathrm{mol} / \mathrm{L}$, 其作用位点同样是 Pks13 的 TE 域(Q1610R、T1645P).

\section{$1.6 \mathrm{MmpL} 3$ 及其抑制剂}

$\mathrm{MmpL}$ 蛋白是一类跨膜转运蛋白, 依赖质子动力转 运底物透过细胞膜 ${ }^{[53]}$. 结核分枝杆菌基因组中包含 13 种 $\mathrm{MmpL}$ 基因 ${ }^{[8]}$, MmpL3 (Rv0206c) 是其中唯一必需 的 ${ }^{[54-55]}$. MmpL3 含有 $800 \sim 1100$ 个氨基酸, 分子量范围

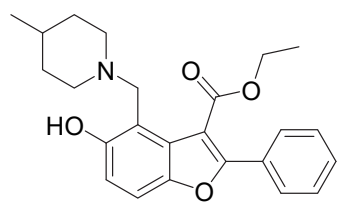

23 TAM1

$\mathrm{IC}_{50}: 0.26 \pm 0.03 \mu \mathrm{mol} / \mathrm{L}$ $\mathrm{MIC}_{\mathrm{H} 37 \mathrm{Rv}}: 2.3 \mu \mathrm{mol} / \mathrm{L}$<smiles>C1CCCCC1</smiles><smiles></smiles>

25 coumestan48 $\mathrm{MIC}_{\mathrm{H} 37 \mathrm{Rv}}: 3.9 \mathrm{ng} / \mathrm{mL}$ $t_{1 / 2}: 120.1 \mathrm{~min}$ Cli human: $0.1 \mathrm{~mL} / \mathrm{min} / \mathrm{g}$

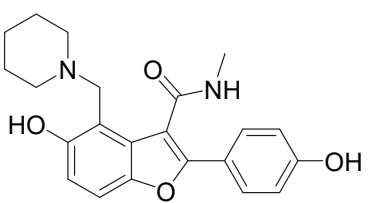

24 TAM16

$\mathrm{IC}_{50}: 0.19 \pm 0.01 \mu \mathrm{mol} / \mathrm{L}$ $\mathrm{MIC}_{\mathrm{H} 37 \mathrm{Rv}}: 0.09 \mu \mathrm{mol} / \mathrm{L}$ $t_{1 / 2}: 60 \mathrm{~min}$

Cli human $<0.5 \mathrm{~mL} / \mathrm{min} / \mathrm{g}$

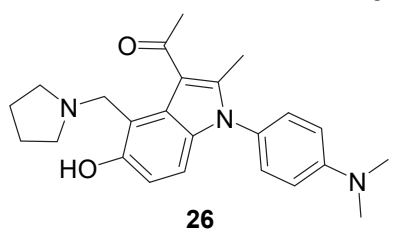

$\mathrm{MIC}_{\mathrm{H} 37 \mathrm{Rv}}: 0.244 \mu \mathrm{mol} / \mathrm{L}$
图式 8 Pks13 抑制剂

Scheme 8 Pks13 inhibitors

表 $4 \mathrm{MmpL} 3$ 抑制剂的 $\mathrm{MIC}_{\mathrm{H} 37 \mathrm{Rv}}$ 值、基因突变以及具体位置

Table 4 MICH37Rv value of MmpL3 inhibitor, gene mutations and specific position

\begin{tabular}{llll}
\hline \multicolumn{1}{c}{ Compound } & \multicolumn{1}{c}{ MIC $_{\mathrm{H} 37 \mathrm{Rv}}$} & \multicolumn{1}{c}{ AA change } & Location \\
\hline AU1235 & $0.01 \mu \mathrm{g} / \mathrm{mL}$ & $\mathrm{G} 253 \mathrm{E}$ & $\mathrm{TMH}^{a} \mathrm{X}$ \\
BM212/BM635 & $0.12 / 0.3 \mu \mathrm{mol} / \mathrm{L}$ & $\mathrm{L} 215 \mathrm{~S}$ & $\mathrm{TMH} \mathrm{IX}$ \\
indole carboxamides & $0.012 \mu \mathrm{mol} / \mathrm{L}$ & $\mathrm{S} 288 \mathrm{~T}$ & $\mathrm{TMH}$ XI \\
HC2091 & $19.3 \mu \mathrm{mol} / \mathrm{L}^{b}$ & $\mathrm{~V} 643, \mathrm{~F} 644$ & $\mathrm{TMH} \mathrm{IV}$ \\
Spiro & $0.06 \mu \mathrm{mol} / \mathrm{L}$ & $\mathrm{F} 255 \mathrm{~L}, \mathrm{Y} 252 \mathrm{C}$ & $\mathrm{TMH}$ X \\
TBL-140 & $2.5 \mu \mathrm{mol} / \mathrm{L}^{b}$ & $\mathrm{~L} 189 \mathrm{R}, \mathrm{L} 567 \mathrm{P}, \mathrm{V} 643 \mathrm{M}, \mathrm{F} 644 \mathrm{~L}$ & $\mathrm{TMH} \mathrm{VIII,} \mathrm{II,} \mathrm{IV}$ \\
THPP & $0.15 \mu \mathrm{mol} / \mathrm{L}$ & A249P, A677V & TMH X, V \\
SQ109 & $0.78 \sim 1.56 \mu \mathrm{mol} / \mathrm{L}$ & A700T, Q40R, L567P & TMH V, VII; 周质区 \\
\hline
\end{tabular}

${ }^{a} \mathrm{TMH}$ : transmembrane $a$ helices, the number comes from the crystal structure (PDB ID: $\left.5 \mathrm{AJF}\right) .{ }^{b} \mathrm{MIC} \mathrm{H}_{\mathrm{H} 7 \mathrm{Rv}}$ : the lowest concentration of drug leading to at least $99 \%$ inhibition of bacterial growth. 
为 $100000 \sim 122000 . \mathrm{MmpL} 3$ 包含 12 个跨膜 $\alpha$ 螺旋, 负 责将细胞质内的 TMM 转运到细胞膜外 ${ }^{[56]}$. 近十年, 人 们通过全细胞䇻选和全基因测序技术得到了很多以 MmpL3 为靶点的抑制剂, 分子结构多种多样, 包括 SQ109、TBL-140、AU1235、BM212、HC2091、吲哚 酰胺类化合物、Spiro 和 THPP ${ }^{[57-68]}$. 表 4 中列出了代表 性的抑制剂，对应的抑菌活性以及基因突变的具体位 置.

SQ109 (27)是一种乙二胺类化合物, 当前处于临床 $\mathrm{IIb} \sim \mathrm{III}$ 期. 改变 27 结构左侧不饱和烷烃链的长度, 用 芳香环、脂肪环替代烷烃链或者还原该不饱和烷烃链等 改造策略, 都会使化合物的抗菌活性出现不同程度的下 降; 用其它环替代右侧金刚烷基时，活性同样降低. 27 对耐药和药物敏感型分枝杆菌都有较强的抑制作用，与 其它抗结核药物联用可有效缩短治疗周期[69-70]. 据研究 发现 27 抑制 MmpL3 可能有三种作用机制: 直接抑制 MmpL3、降低质子动势、以及抑制甲菜醌的生物合成 ${ }^{[60]}$. 27 较低的致突变频率 $\left(\sim 2.55 \times 10^{-11}\right)$ 可能与它作用机制 的多样性有关. 另外还有一些 27 类似物作为 MmpL3 抑 制剂正在被研究, 如 TBL-140 (28), 它有着更好的代谢 稳定性 ${ }^{[64]}$ (Scheme 9).

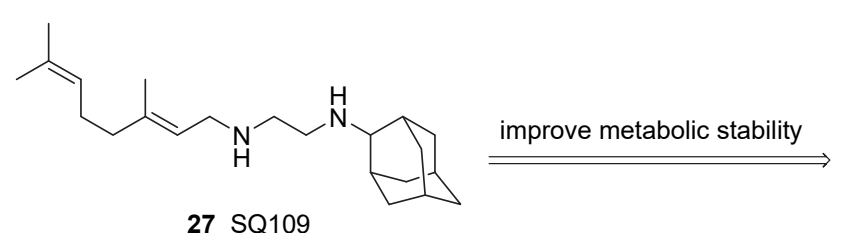

$\mathrm{MIC}_{\mathrm{H} 37 \mathrm{Rv}}: 0.78 \sim 1.56 \mu \mathrm{mol} / \mathrm{L}$ HLM stability $t_{1 / 2}: 2.97 \mathrm{~min}$ Cli human: $0.3 \mathrm{~mL} / \mathrm{min} / \mathrm{g}$<smiles>FC(F)(F)Oc1ccc(Oc2ccc(CNCCNC3C4CC5CC(C4)CC3C5)cc2)cc1</smiles>

28 TBL-140

$\mathrm{MIC}_{99}$, H37Rv: $2.5 \mu \mathrm{mol} / \mathrm{L}$ HLM stability $t_{1 / 2}: 231 \mathrm{~min}$ Cli human: $0.003 \mathrm{~mL} / \mathrm{min} / \mathrm{g}$

图式 9 乙二胺类 MmpL3 抑制剂 Scheme 9 Ethylenediamines MmpL3 inhibitors

2013 年 Ballell 等 ${ }^{[71]}$ 篮选得到吲哚酰胺类化合物 29 对结核分枝杆菌有抑制作用. Kozikowski 课题组 ${ }^{[61,72-73]}$ 用苯环或含氮杂环替代环己基，活性降低; 并且发现吲 哚-2-酰胺母核中两个 $\mathrm{NH}$ 基团为活性必需. 为进一步提 升细胞水平活性, 将 $\mathbf{2 9}$ 优化为 $\mathbf{3 0}, \mathbf{3 0}$ 对 $\mathrm{H} 37 \mathrm{Rv}$ 菌株的 抑制活性为 $0.012 \mu \mathrm{mol} / \mathrm{L}$, 同时对 $\mathrm{H} 37 \mathrm{Rv} / \mathrm{DS}$ 、 V4207/DS、KZN494/MDR、V2475/MDR、TF274/XDR、 R506/XDR 这六种菌株的抑制作用分别达到 0.012 、 $0.023 、 0.012 、 0.047 、 0.006$ 和 $0.012 \mu \mathrm{mol} / \mathrm{L}^{[74]}$. 在 ADMET
性质上, 30 的代谢稳定性较好, 细胞毒性较小 (Vero $\mathrm{IC}_{50}>192 \mu \mathrm{mol} / \mathrm{L}$ ), 对 CYP450 各亚型和 $\mathrm{hERG}$ 钾通道 无明显抑制作用, 在小鼠模型中以 $100 \mathrm{mg} / \mathrm{kg}$ 的剂量口 服给药, 30 在小鼠肺部和血浆中达到最大浓度的时间都 为 $4 h^{[74]}$ (表 5). 另外, 30 与低剂量利福平(RIF)联用有很 好的协同作 用 ${ }^{[75]}$. 同时，诺华公司为改善苗头化合物 的代谢稳定性，将其优化为 NITD-304 (31) 和 NITD-349 (32). 在 CD-1 小鼠的感染模型中以 $25 \mathrm{mg} / \mathrm{kg}$ 的剂量口 服给药 31 和 32 , 它们的生物利用度分别为 $53 \%$ 和 $51 \%{ }^{[62]}$ (Scheme 10). 在毒性上, 31 和 32 对 HepG2 和 THP-1 细胞的 $\mathrm{CC}_{50}$ 都大于 $20 \mu \mathrm{mol} / \mathrm{L}$, 对 $\mathrm{hERG}$ 钾通道 也无明显抑制作用 $\left(\mathrm{IC}_{50}>30 \mu \mathrm{mol} / \mathrm{L}\right)^{[62]}$ (表 6). 目前吲哚 酰胺衍生物处于临床前研究中.
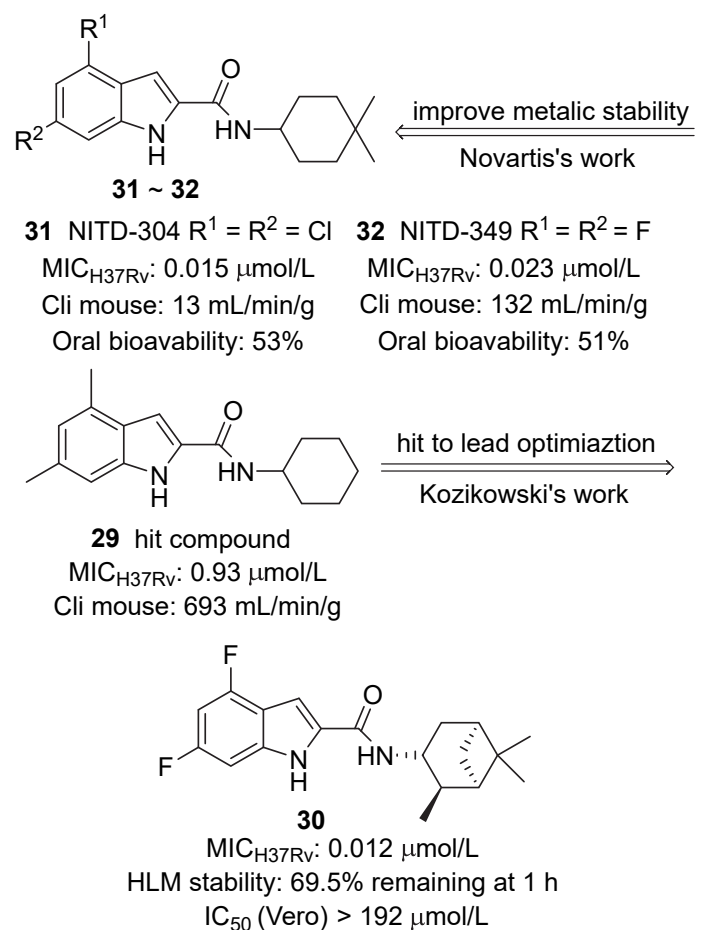

图式 10 吲哚酰胺类 MmpL3 抑制剂 Scheme 10 Indoleamide series of MmpL3 inhibitors

表 5 Compound $\mathbf{3 0}$ 的已报道 ADMET 性质

Table 5 Reported ADMET properties of compound $\mathbf{3 0}$

\begin{tabular}{|c|c|}
\hline Vero cells $\mathrm{IC}_{50}$ & $\geqslant 192 \mu \mathrm{mol} / \mathrm{L}$ \\
\hline Remaining ( $T=120 \mathrm{~min})$ & $52 \%$ \\
\hline CYP inhibition (\% inhibition) CYP2C9 & -5.2 \\
\hline CYP inhibition (\% inhibition) CYP2D6 & 36.6 \\
\hline CYP inhibition (\% inhibition) CY3A4 & -6.7 \\
\hline hERG IC 50 & $>30 \mu \mathrm{mol} / \mathrm{L}$ \\
\hline \multirow[t]{2}{*}{$T_{\max }(100 \mathrm{mg} / \mathrm{kg}$ po $)$} & Plasma $4 \mathrm{~h}$ \\
\hline & Lung $4 \mathrm{~h}$ \\
\hline \multirow[t]{2}{*}{$c_{\max }(100 \mathrm{mg} / \mathrm{kg}$ po $)$} & Plasma $1.71 \mu \mathrm{g} / \mathrm{unit}^{a}$ \\
\hline & Lung $3.50 \mu \mathrm{g} / \mathrm{unit}$ \\
\hline
\end{tabular}


BM212 (33)，33 与 27 的作用机制类似，直接抑制 MmpL3 转运蛋白的同时还抑制 TMM 的转运过程. 为提 高 33 的细胞水平活性, 他们在初步结构改造中发现了 BM635 (34), 其活性将近提高 40 倍. 由于 34 的水溶性 较差 $(<1 \mu \mathrm{mol} / \mathrm{L})$, Poce 等 ${ }^{[76]}$ 将吡咯氮原子上的 4-氟苯 基替换为异丙基 $(35)$ 后 $\mathrm{MIC}_{\mathrm{H} 37 \mathrm{Rv}}$ 值为 $0.15 \mu \mathrm{mol} / \mathrm{L}$, 水溶 性大幅提升, 且小鼠肝微粒体代谢稳定性明显优于 34 (Scheme 11). 在 C57 小鼠的感染模型中将 35 以 50 $\mathrm{mg} / \mathrm{kg}$ 的剂量持续口服给药 $8 \mathrm{~d}$ 后, 小鼠肺部减少的菌 落数量为 1.5 个 $\log \mathrm{CFU}$.

表 6 NITD-304 和 NITD-349 的已报道 ADMET 性质 Table 6 Reported ADMET properties of NITD-304 and NITD-349

\begin{tabular}{lll}
\hline \multicolumn{1}{c}{ ADMET properties } & NITD-304 & NITD-349 \\
\hline$t_{1 / 2}(25 \mathrm{mg} / \mathrm{kg}$ po in mice $)$ & $233 \mathrm{~min}$ & $11 \mathrm{~min}$ \\
Cytotoxicity HepG2 CC 50 & $>20 \mu \mathrm{mol} / \mathrm{L}$ & $>20 \mu \mathrm{mol} / \mathrm{L}$ \\
Cytotoxicity THP-1 CC 50 & $>20 \mu \mathrm{mol} / \mathrm{L}$ & $>20 \mu \mathrm{mol} / \mathrm{L}$ \\
hERG IC50 & $>30 \mu \mathrm{mol} / \mathrm{L}$ & $>30 \mu \mathrm{mol} / \mathrm{L}$ \\
Cardiovascular Profile & $>30 \mu \mathrm{mol} / \mathrm{L}$ & $\mathrm{NA}^{a}$ \\
QPatch IC 50 & Negative & Negative \\
Ames test & $>20 \mu \mathrm{mol} / \mathrm{L}$ \\
CYP450 inhibition 3A4 IC 50 & $>20 \mu \mathrm{mol} / \mathrm{L}$ & $>20 \mu \mathrm{mol} / \mathrm{L}$ \\
CYP450 inhibition 2D6 IC50 & $>20 \mu \mathrm{mol} / \mathrm{L}$ & $>2.67 \mu \mathrm{mol} / \mathrm{L}$ \\
CYP450 inhibition 2C9 IC50 & $>20 \mu \mathrm{mol} / \mathrm{L}$ & $2.67 \mu \mathrm{mol} / \mathrm{L}$ \\
$c_{\text {max }}(25 \mathrm{mg} / \mathrm{kg}$ po in mice $)$ & $3.51 \mu \mathrm{mol} / \mathrm{L}$ & $4.21 \mu$ \\
\hline
\end{tabular}

${ }^{a}$ NA: Not known.

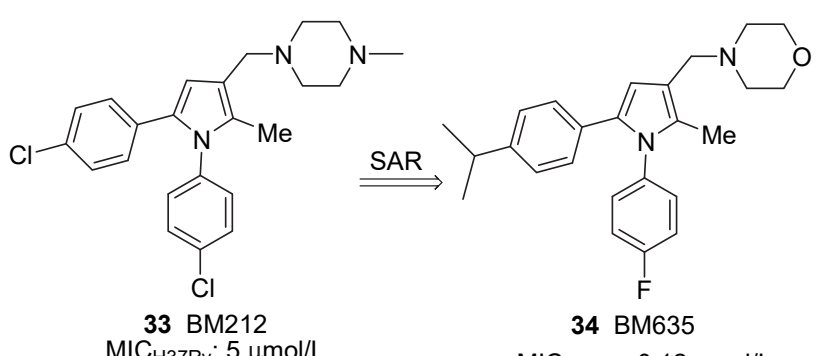

$\mathrm{MIC}_{\mathrm{H} 37 \mathrm{Rv}}: 5 \mu \mathrm{mol} / \mathrm{L}$ Cli mouse: $0.7 \pm 0.1 \mathrm{~mL} / \mathrm{min} / \mathrm{g}$

$\mathrm{MIC}_{\mathrm{H} 37 \mathrm{Rv}}: 0.12 \mu \mathrm{mol} / \mathrm{L}$ Cli mouse: $1.4 \mathrm{~mL} / \mathrm{min} / \mathrm{g}$ Water solubility $<1 \mu \mathrm{mol} / \mathrm{L}$

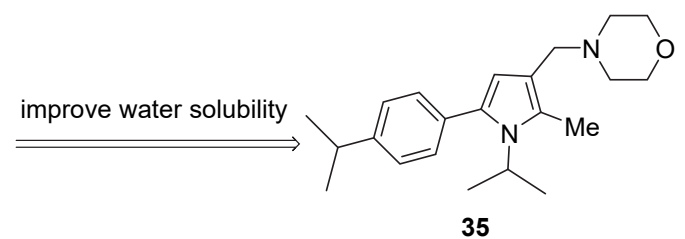

$\mathrm{MIC}_{\mathrm{H} 37 \mathrm{Rv}}: 0.15 \mu \mathrm{mol} / \mathrm{L}$ Cli mouse: $0.02 \mathrm{~mL} / \mathrm{min} / \mathrm{g}$ Water solubility: $199 \mu \mathrm{mol} / \mathrm{L}$

图式 11 BM212 及其类似物的结构

Scheme 11 Structures of BM212 and its analogues

螺环类化合物是由 GSK 公司发现的. 为改善 36 的
代谢稳定性, Remuinan 等将 ${ }^{[63]}$ 其优化为 37 (Scheme 12), 后者在小鼠肝微粒中清除率为 $1.2 \mathrm{~mL} / \mathrm{min} / \mathrm{g}$. 金刚烷艮 类化合物 AU1235 (38)在 2012 年由 Scherman 等[77]䇻选 发现, 38 能使 RIF 在 $M . t b$ 中积聚并且在体外与 RIF 和 贝达喹啉有协同抑菌效应. 但在类药性评价中发现 38 及其类似物对人类可溶性环氧化物水解酶 $(\mathrm{sEH})$ 有显著 的抑制作用 $\left(\mathrm{IC}_{50}: 0.4 \mathrm{nmol} / \mathrm{L}\right)$, 与血浆蛋白的结合率也 非常高(98.8\%)，这些不良因素限制了该类化合物的进 一步发展

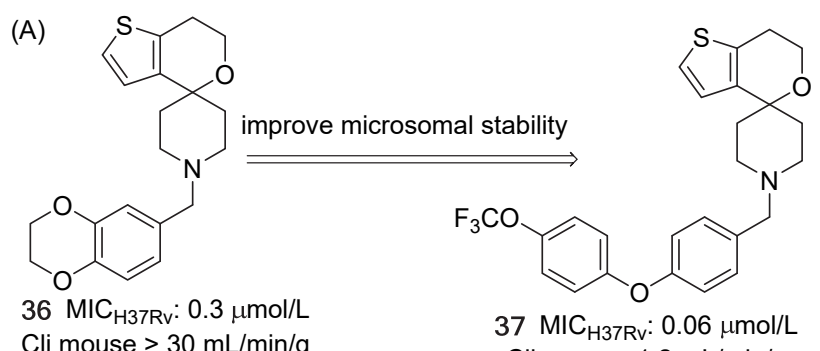

$\begin{array}{rr}\text { Cli mouse }>30 \mathrm{~mL} / \mathrm{min} / \mathrm{g} & 37 \mathrm{MIC}_{\mathrm{H} 37 \mathrm{Rv}}: 0.06 \mu \mathrm{mol} / \mathrm{L} \\ & \text { Cli mouse: } 1.2 \mathrm{~mL} / \mathrm{min} / \mathrm{g}\end{array}$

(B)

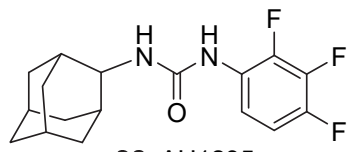

38 AU1235

$\mathrm{MIC}_{\mathrm{H} 37 \mathrm{Rv}}: 0.01 \mu \mathrm{g} / \mathrm{mL}$

图 12 (A)提高螺环类化合物的代谢稳定性和(B) AU1235 的 结构

Scheme 12 (A) Improve the metabolic stability of spiros, and (B) structure of AU1235

四氢吡唑并嘧啶类化合物 39 由于半衰期短、血浆 分布率低，导致其在动物实验中无活性 ${ }^{[63]}$. Zheng 等 ${ }^{[67]}$ 于 2018 年笁选得到 HC2091 (40)对 H37Rv 菌株的 MIC 99 值为 $19.3 \mu \mathrm{mol} / \mathrm{L}$. 他们在构效关系研究中发现 $\mathbf{4 0}$ 的酰 胺部分与苯环间的两个碳原子为活性必需，当苯环上的 氯原子被异丁基、乙基等基团取代时，活性明显提升; 当苯环的其它位置有取代基时，活性显著降低. 利莫那 班(rimonabant, 41)与 33 具有相似的分子结构, 因此 41 对 H37Rv 菌株也有一定的抑制作用, MIC 值为 25 $\mu \mathrm{g} / \mathrm{mL}^{[78]}$.

2019 年, 饶子和院士课题组 ${ }^{[79]}$ 解析出 MmpL3 的晶 体结构, 提出了 MmpL3 抑制剂(AU1235, SQ109, 吲哚 酰胺类，利莫那班)的作用机制: 小分子插入质子转移 通道中，使两对关键氨基酸作用力断裂(Asp256-Tyr646, Asp645-Tyr257), 导致质子动力降低, 进而抑制 MmpL3. 在靶点确证上, $\mathrm{Li}$ 等 ${ }^{[80]}$ 用吲哚酰胺类化合物与荧光基团 共价结合形成一种分子探针 North100 (42), 可鉴别该靶 点的抑制剂是否直接抑制 MmpL3 转运蛋白(Scheme $13)$. 


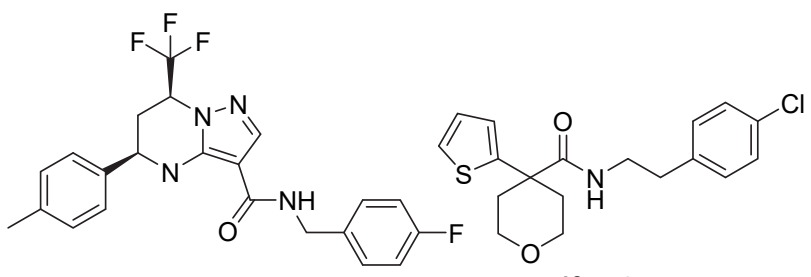

39 THPP

$\mathrm{MIC}_{\mathrm{H} 37 \mathrm{Rv}}: 0.15 \mu \mathrm{mol} / \mathrm{L}$

$40 \mathrm{HC} 2091$

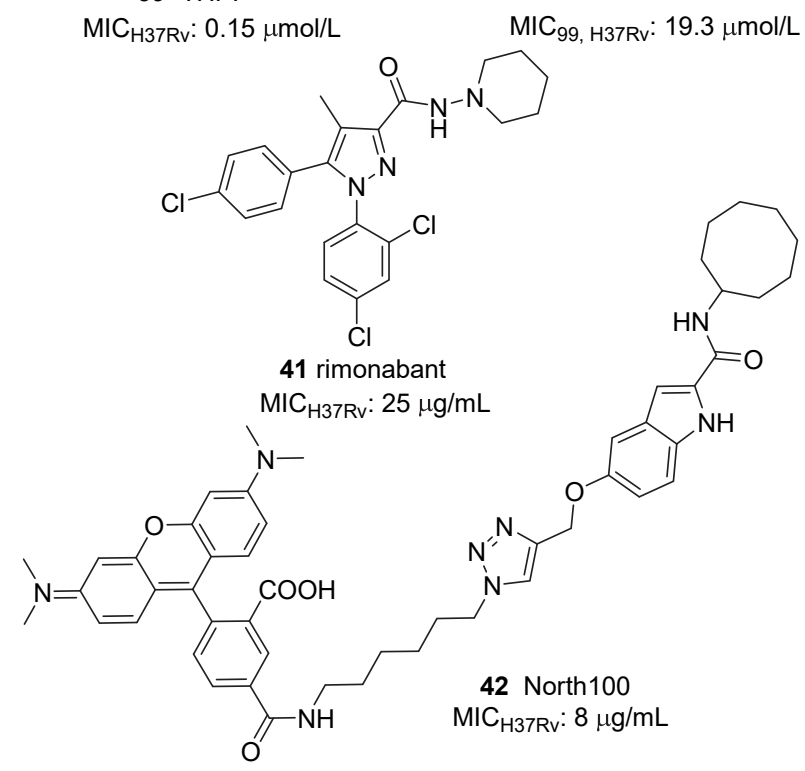

图式 13 其他类 MmpL3 抑制剂

Scheme 13 Other MmpL3 inhibitors

\section{$1.7 \mathrm{Ag} 85$ 蛋白及其抑制剂}

Ag85 蛋白家族包含 Ag85A (PDB ID: 1SFR)、Ag85B (PDB ID: 1F0N)、Ag85C (PDB ID: 1VA5), 这三个同源 蛋白的晶体结构分别于 2001 年、 2004 年相继被解析出 来 ${ }^{[81-82]} . \mathrm{Ag} 85 \mathrm{ABC}$ 属于 $\alpha / \beta$ 水解酶, 分别由 $304 、 285$ 、 303 个氨基酸组成，它们之间有 $70.8 \% \sim 77.5 \%$ 的同源 性 ${ }^{[83]}$. Ag85 蛋白在 $M . t b$ 中负责与丝氨酸水解酶协同将 $\mathrm{TMM}$ 上的分枝菌酸链转移到细胞壁上 ${ }^{[84]}$. Warrier 等 ${ }^{[85]}$ 发现一类 $\mathrm{Ag} 85 \mathrm{C}$ 蛋白抑制剂(43)在普通菌株和耐药菌株 中均表现出良好的活性. 43 与 $\mathrm{Ag} 85 \mathrm{C}$ 的共晶结构显示该 分子的噻吩环在空间上阻碍了 $\mathrm{Ag} 85 \mathrm{C}$ 与 $\mathrm{TMM} \alpha$ 链的结 合, 从而阻断了 $M . t b$ 细胞壁上重要脂质的合成. Lehmann 等 ${ }^{[86]}$ 利用 $\beta$-内酯(44)模拟分枝菌酸结构, 从而阻断 了分枝菌酸合成过程中丝氨酸水解酶、Ag85 与分枝菌 酸酯的结合, 进而抑制细胞壁的合成(Scheme 14).

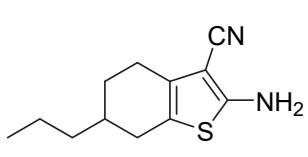

$43 \mathrm{I}_{3}-\mathrm{AG}_{85}$ $\mathrm{MIC}_{\mathrm{H} 37 \mathrm{Rv}}: 0.5 \sim 1.0 \mu \mathrm{mol} / \mathrm{L}$

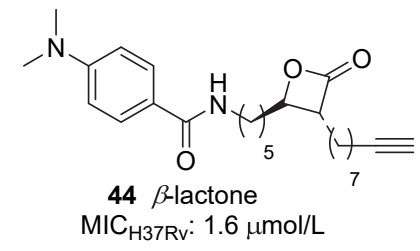

图式 14 Ag85 抑制剂

Scheme 14 Ag85 inhibitors

\section{8 未知靶点的抑制剂}

2014 年被欧盟批准上市的德拉马尼(Delamanid, 45) 属于硝基味唑衍生物，需要经过硝基还原酶活化后抑制 分枝菌酸的合成 ${ }^{[20]}$. 在靶点认证实验中, 对抗性突变体 进行全基因测序, 突变位点位于编码与 45 活化过程相 关的酶上, 所以 45 的具体靶点仍未确认 ${ }^{[87]} .45$ 源于 1989 年 Ciba-Geigy 公司报道的化合物 CGI-17341 (46) ${ }^{[88]}$, 其致突变作用限制了它的发展, Matsumoto 等 ${ }^{[89]}$ 将侧链的 2 位替换为氧原子改善了致突变问题. 基于前 期研究, Sasaki 等 ${ }^{[00]}$ 为改善水溶性以提高生物利用度, 在苯环对位引入哌啶得到 47; 为寻找细胞水平和体内 活性更好的分子, 在进一步结构改造中发现了德拉马尼 (Scheme 15).
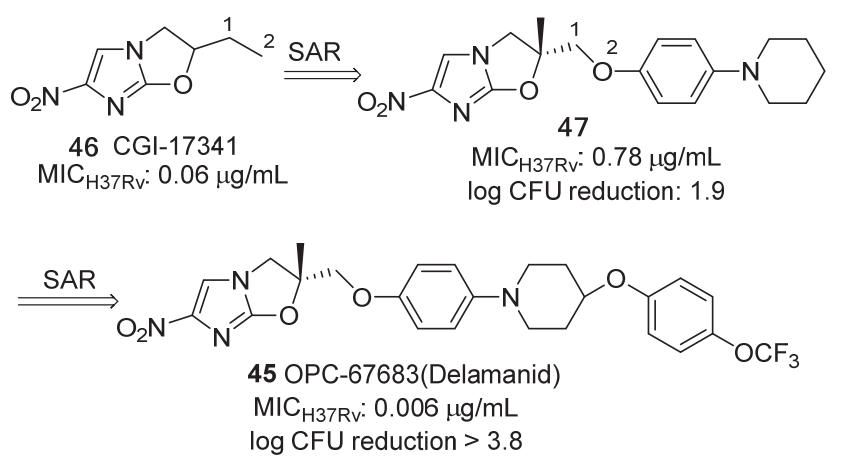

图式 15 德拉马尼的发现

Scheme 15 Discovery of Delamanid

北京协和医学院的张东峰课题组将 $\mathbf{2 5}$ 的苯并呋喃 母核替换为苯并吡喃酮得到了化合物 48 (Scheme 16), 48 具有良好的肝微粒体稳定性. 在 BALB $/ \mathrm{c}$ 小鼠急性感 染模型中, 48 能有效降低肺部菌落数量 ${ }^{[91]}$. 然而该类化 合物未进行靶点确证.

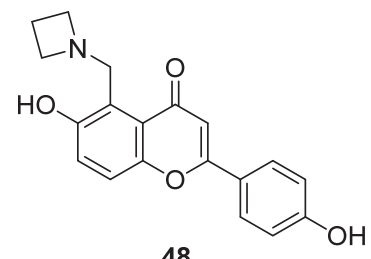

$\mathrm{MIC}_{\mathrm{H} 37 \mathrm{Rv}}=0.45 \mu \mathrm{g} / \mathrm{mL}$

MLM stability: $96 \%$ remaining after $30 \mathrm{~min}$

图式 16 苯并吡喃酮衍生物

Scheme 16 4H-Chromen-4-one derivates

\section{2 结论与展望}

虽然近半个世纪以来仅有贝达喹啉和硝基咪唑两 类抗结核新化学实体被批准上市, 但已对严重耐药结核 病的治疗带来巨大福音. 据 2020 年新英格兰杂志报道, 对严重耐药结核病患者采用贝达喹啉、普托马尼和利奈 
唑胺三药联用的治疗方案可以极大缩短治疗周期至 6 个

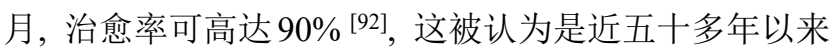
结核病治疗的重大突破之一. 异烟肼已被人们使用将近 七十年, 现在仍然作为一线药物来治疗各类型的结核 病, 这说明了基于分枝菌酸的生物合成和转运途径研发 的药物有其独特的优势. 同时近年来越来越多靶向此途 径的化合物处于临床及临床前研究的各个阶段(表 7),
SQ109 在I期实验中已经显示出优良的安全性、耐受性 和显著的疗效，当前处于临床 IIb III期; 吲唑磺酰胺 类、噻二唑类和吲哚酰胺类化合物显示出优异的体内/ 外活性，目前已进入临床前研究中. 靶向分枝菌酸生物 合成和转运途径的抗结核新化学实体的发现与发展有 望为耐药结核病的治疗提供更多选择.

表 7 靶向分枝菌酸的代表性抗结核抑制剂

Table 7 Representative antitubercular inhibitors targeting to mycolic acid

\begin{tabular}{llll}
\hline 靶点 & 代表性小分子化合物 & 结构类别 & 发展阶段 \\
\hline KasA & DG167, JSF-3285 & 吲唑磺酰胺类 & Lead Optimization \\
InhA & GSK693 & 噻二唑类 & Lead Optimization \\
& NITD916 & 4-羟基-2-吡啶酮类 & Hit-to-Lead \\
HadABC & PT03 & 二芳基醚类 & Hit-to-Lead \\
FadD32 & NAS21 & 1,3-二酮类 & Hit-to-Lead \\
Pks13 & 喹啉-2-酰胺 & 喹啉类 & Hit-to-Lead \\
& TP4 & 噻吩类 & Hit-to-Lead \\
MmpL3 & TAM16, Coumestan48 & 苯并呋喃类 & Lead Optimization \\
& SQ109 & 乙二胺类 & Phase IIb III \\
& NITD-304, NITD-349 & 吲哚酰胺类 & Pre-Clinical \\
& AU1235 & 金刚烷脲类 & Hit-to-Lead \\
& BM212/BM635 & 吡咯和吡唑类 & Hit-to-Lead \\
& THPP & 四氢吡唑并 $[1,5-a]$ 嘧啶类 & Hit-to-Lead \\
Ag85 & Spiro & 哌啶螺环类 & Hit-to-Lead \\
未知靶点 & PIPD1 & 差基哌啶类 & Hit-to-Lead \\
\hline & I3-AG85 德拉马尼 & 塞吩类 & Hit-to-Lead \\
& & 亚硝基咪唑类 & EMA 上市
\end{tabular}

\section{References}

[1] WHO Global Tuberculosis Report 2020, World Health Organization, 2020. https://www.who.int/tb/publications/global_report/zh/.

[2] Zumla, A. I.; Gillespie, S. H.; Hoelscher, M.; Philips, P. P.; Cole, S. T.; Abubakar, I.; McHugh, T. D.; Schito, M.; Maeurer, M.; Nunn, A. J. Lancet Infect. Dis. 2014, 14, 327.

[3] Ivanyi, J. Tuberc. Pathog. Prot. Control 1994, 437.

[4] Sun, P.; Mei, J. Chin. J. Antituberc. 2010, 43, 44 (in Chinese). (孙丕, 梅建, 中国防㾤杂志, 2010, 43, 44.)

[5] Jackson, M.; Stadthagen, G.; Gicquel, B. Tuberculosis 2007, 87, 78.

[6] Yuan, Y.; Lee, R. E.; Besra, G. S.; Belisle, J. T.; Barry III, C. E. Proc. Natl. Acad. Sci. U. S. A. 1995, 92, 6630.

[7] North, E. J.; Jackson, M.; Lee, R. E. Curr. Pharm. Design 2014, 20, 4357.

[8] Cole, S. T.; Brosch, R.; Parkhill, J.; Garnier, T.; Churcher, C.; Harris ,D.; Gordon S. V.; Eiglmeier, K.; Gas, S.; Barry, C. E.; Tekaia, F.; Badcock, K.; Basham, D.; Brown, D.; Chillingworth, T.; Connor, R.; Davies, R.; Devlin, K.; Feltwell, T.; Gentles, S.; Hamlin, N.; Holroyd, S.; Hornsby, T.; Jagels, K.; Krogh, A.; McLean, J.; Moule, S.; Murphy, L.; Oliver, K.; Osborne, J.; Quail, M. A.; Rajandream, M. A.; Rogers, J.; Rutter, S.; Seeger, K.; Skelton, J.; Squares, R.; Squares, S.; Sulston, J. E.; Taylor, K.; Whitehead, S.; Barrell, B. G. Nature 1998, 393, 537.

[9] Daffé, M.; Draper, P. Adv. Microb. Physiol. 1998, 39, 131.
[10] Marrakchi, H.; Lanéelle, M. A.; Daffé, M. Chem. Biol. 2014, 21, 67.

[11] Takayama, K; Wang, C; Besra, G. S. Clin. Microbiol. Rev. 2005 18,81 .

[12] Kremer, L.; Nampoothiri, K. M.; Lesjean, S.; Dover, L. G.; Graham, S.; Betts, J.; Brennan, P. J.; Minnikin, D. E.; Locht, C.; Besra, G. S. J. Biol. Chem. 2001, 276, 27967.

[13] Choi, K. H.; Kremer, L.; Besra, G. S.; Rock, C. O. J. Biol. Chem. 2000, 275, 28201.

[14] Kremer, L.; Dover, L. G.; Carrère, S.; Nampoothiri, K. M.; Lesjean, S.; Brown, A. K.; Brennan, P. J.; Minnikin, D. E.; Locht, C.; Besra, G. S. Biochem. J. 2002, 364, 423.

[15] Marrakchi, H.; Ducasse, S.; Labesse, G.; Montrozier, H.; Margeat, E.; Emorine, L.; Charpentier, X.; Daffé, M.; Quemard, A. Microbiology 2002, 148, 951.

[16] Banerjee A, Dubnau E, Quemard A, Balasubramanian, V.; Um, K. S.; Wilson, T.; Collins, D.; Lisle, G. D.; Jacobs, W. R. Jr. Science 1994, 263227.

[17] Odriozola, J. M.; Ramos, J. A.; Bloch, K. Biochim. Biophys. Acta, Lipids Lipid Metab. 1977, 488, 207.

[18] Trivedi, O. A; Arora, P.; Sridharan, V.; Tickoo, R.; Mohanty, D.; Gokhale, R. S. Nature 2004, 428, 441.

[19] Bhatt, A.; Brown, A. K.; Singh, A.; Minnikin, D. E.; Besra, G. S. Chem. Biol. 2008, 15, 930.

[20] Lea-Smith, D. J; Pyke, J. S; Tull, D; McConville, M. J., Coppel, R. L.; Crellin, P. K. J. Biol. Chem. 2007, 282, 11000. 
[21] Grzegorzewicz, A. E.; Pham, H.; Gundi, V. A. K. B.; Scherman, M. S.; North, E. J.; Hess, T.; Jones, V.; Gruppo, V.; Born, S. E. M.; Kordulakova, J.; Chavadi, S. S.; Morisseau, C.; Lenaerts, A. J.; Lee, R. E.; McNeil, M. R.; Jackson, M. Nat. Chem. Biol. 2012, 8, 334.

[22] Jackson, M.; Raynaud, C.; Laneelle, M. A.; Guilhot, C.; Laurent-Winter, C.; Ensergueix, D.; Gicquel, B.; Daffe, M. Mol. Microbiol. 1999, 31, 1573.

[23] Kalscheuer, R.; Weinrick, B.; Veeraraghavan, U.; Besra, G. S.; Jacobs, W. R. Jr. Proc. Natl. Acad. Sci. U. S. A. 2010, 107, 21761.

[24] Ramaswamy, S. V.; Reich, R.; Dou, S. J.; Jasperse, L.; Pan, X.; Wanger, A.; Quitugua, T.; Graviss, E. A. Antimicrob. Agents Chemother. 2003, 47, 1241.

[25] DeBarber, A. E.; Mdluli, K.; Bosman, M.; Bekker, L. G.; Barry, C. E. Proc. Natl. Acad. Sci. U. S. A. 2000, 97, 9677.

[26] Liu, Y. G.; Matsumoto, M.; Ishida, H.; Ohguro, K.; Yoshitake, M.; Gupta, R.; Geiter, L.; Hafkin, J. Tuberculosis 2018, 111, 20.

[27] Kumar, P.; Capodagli, G. C.; Awasthi, D.; Shrestha, R.; Maharaja, K.; Sukheja, P.; Li, S. G.; Inoyama, D.; Zimmerman, M.; Liang, H. P. H.; Sarathy, J.; Mina, M.; Rasic, G.; Russo, R.; Perryman, A.L.; Richmann, T.; Gupta, A.; Singleton, E.; Verma, S.; Husain, S.; Soteropoulos, P.; Wang, Z.; Morris, R.; Porter, G.; Agnihotri, G.; Salgame, P.; Ekins, S.; Rhee, K. Y.; Connell, N.; Dartois, V.; Neiditch, M. B.; Freundlich, J. S.; Alland, D. mBio 2018, 9, e02101.

[28] Abrahams, K. A.; Chung, C. W.; Ghidelli-Disse, S.; Rullas, J.; Rebollo-Lopez, M. J.; Gurcha, S. S.; Cox, J. A. G.; Mendoza, A.; Jimenez-Navarro, E.; Martinez-Martinez, M. S.; Neu, M.; Shillings, A.; Homes, P.; Argyrou, A.; Casanueva, R.; Loman, N. J.; Moynihan, P. J.; Lelievre, J.; Selenski, C.; Axtman, M.; Kremer, L.; Bantscheff, M.; Angulo-Barturen, I.; Izquierdo, M. C.; Cammack, N. C.; Drewes, G.; Ballell, L.; Barros, D.; Besra, G. S.; Bates, R. H. Nat. Commun. 2016, 7, 12581 .

[29] Inoyama, D.; Awasthi, D.; Capodagli, G. C.; Tsotetsi, K.; Sukheja, P.; Zimmerman, M.; Li, S. G.; Jadhav, R.; Russo, R.; Wang, X.; Grady, C.; Richmann, T.; Shrestha, R.; Li, L. P.; Ahn, Y. M.; Ho, L. H. P.; Mina, M.; Park, S.; Freundlich, J. S. Cell Chem. Biol. 2020, 27, 560-570.

[30] Chollet, A.; Mourey, L.; Lherbet, C.; Delbot, A.; Julien, S.; Baltas, M.; Bernadou, J.; Pratviel, G.; Maveyraud, L.; Bernardes-Génisson, V. J. Struct. Biol. 2015, 190, 328.

[31] Rozwarski, D. A.; Vilcheze, C.; Sugantino, M.; Bittman, R.; Sacchettini, J. C. J. Biol. Chem. 1999, 274, 15582.

[32] Chollet, A.; Mourey, L.; Lherbet, C.; Delbot, A.; Julien, S.; Baltas, M.; Bernadou, J.; Pratviel, G.; Maveyraud, L.; Bernardes-Génisson, V. J. Struct. Biol. 2015, 190, 328.

[33] Martinez-Hoyos, M.; Perez-Herran, E.; Gulten, G.; Encinas, L.; Alvarez-Gomez, D.; Alvarez, E.; Ferrer-Bazaga, S.; Garcia-Perez, A.; Ortega, F.; Angulo-Barturen, I.; Rullas-Trincado, J.; Blanco Ruano, D.; Torres, P.; Castaneda, P.; Huss, S.; Fernandez Menendez, R.; Gonzalez Del Valle, S.; Ballell, L.; Barros, D.; Modha, S.; Dhar, N.; Signorino-Gelo, F.; McKinney, J. D.; Garcia-Bustos, J. F.; Lavandera, J. L.; Sacchettini, J. C.; Jimenez, M. S.; Martin-Casabona, N.; Castro-Pichel, J.; Mendoza-Losana, A. EBioMedicine 2016, 8, 291.

[34] Hartkoorn, R. C.; Sala, C.; Neres, J.; Pojer, F.; Magnet, S.; Mukherjee, R.; Uplekar, S.; Boy-Rottger, S.; Altmann, K. H.; Cole, S. T. EMBO Mol. Med. 2012, 4, 1032.

[35] Manjunatha, U. H.; Rao, S. P. S.; Kondreddi, R. R.; Noble, C. G.; Camacho, L. R.; Tan, B. H.; Ng, S. H.; Ng, P. S.; Ma, N. L.; Lakshminarayana, S. B.; Herve, M.; Barnes, S. W.; Yu, W. X.; Kuhen, K.; Blasco, F.; Beer, D.; Walker, J. R.; Tonge, P. J.; Glynne, R.; Smith, P. W.; Diagana, T. T. Sci. Transl. Med. 2015, 7, 1.

[36] Pan, P.; Tonge, P. J. Curr. Top. Med. Chem. 2012, 12, 672.

[37] Dong, Y.; Qiu, X.; Shaw, N.; Xu, Y.; Sun, Y.; Li, X.; Li, J.; Rao, Z. Protein Cell. 2015, 6, 504.

[38] Bhowruth, V.; Brown, A. K.; Besra, G. S. Microbiology 2008, 154, 1866.

[39] Grzegorzewicz, A. E.; Kordulakova, J.; Jones, V.; Born, S. E.; Belardinelli, J. M.; Vaquie, A.; Gundi, V. A.; Madacki, J.; Slama, N.;
Laval, F.; Vaubourgeix, J.; Crew, R. M.; Gicquel, B.; Daffe, M.; Morbidoni, H. R.; Brennan, P. J.; Quemard, A.; McNeil, M. R.; Jackson, M. J. Biol. Chem. 2012, 287, 38434.

[40] Portevin, D.; de Sousa-D'Auria, C.; Montrozier, H.; Houssin, C.; Stella, A.; Laneelle, M. A.; Bardou, F.; Guilhot, C.; Daffe, M. J. Biol. Chem. 2005, 280, 8862 .

[41] Kuhn, M. L.; Alexander, E.; Minasov, G.; Page, H. J.; Warwrzak, Z.; Shuvalova, L.; Flores, K. J.; Wilson, D. J.; Shi, C.; Aldrich, C. C.; Anderson, W. F. ACS Infect. Dis. 2016, 2, 579.

[42] Stanley, S. A.; Kawate, T.; Iwase, N.; Shimizu, M.; Clatworthy, A. E.; Kazyanskaya, E.; Sacchettini, J. C.; Ioerger, T. R.; Siddiqi, N. A.; Minami, S.; Aquadro, J. A.; Grant, S. S.; Rubin, E. J.; Hung, D. T. Proc. Natl. Acad. Sci. U. S. A. 2013, 110, 11565.

[43] Fang, C.; Lee, K. K.; Nietupski, R.; Bates, R. H.; Fernandez-Menendez, R.; Lopez-Roman, E. M.; Guijarro-Lopez, L.; Yin, Y.; Peng, Z.; Gomez, J. E.; Fisher, S.; Barros-Aguirre, D.; Hubbard, B. K.; Serrano-Wu, M. H.; Hung, D. T. Bioorg. Med. Chem. Lett. 2018, 28, 3529.

[44] Galandrin, S.; Guillet, V.; Rane, R. S.; Leger, M.; N, R.; Eynard, N.; Das, K.; Balganesh, T. S.; Mourey, L.; Daffe, M.; Marrakchi, H. J. Biomol. Screen 2013, 18, 576.

[45] Bergeret, F.; Gavalda, S.; Chalut, C.; Malaga, W.; Quemard, A.; Pedelacq, J. D.; Daffe, M.; Guilhot, C.; Mourey, L.; Bon, C. J. Biol. Chem. 2012, 287, 33675.

[46] Wilson, R.; Kumar, P.; Parashar, V.; Vilcheze, C.; Veyron-Churlet, R.; Freundlich, J. S.; Barnes, S. W.; Walker, J. R.; Szymonifka, M. J.; Marchiano, E.; Shenai, S.; Colangeli, R.; Jacobs, W. R., Jr.; Neiditch, M. B.; Kremer, L.; Alland, D. Nat. Chem. Biol. 2013, 9, 499.

[47] Thanna, S.; Knudson, S. E.; Grzegorzewicz, A.; Kapil, S.; Goins, C. M.; Ronning, D. R.; Jackson, M.; Slayden, R. A.; Sucheck, S. J. Org. Biomol. Chem. 2016, 14, 6119.

[48] Ioerger, T. R.; O'Malley, T.; Liao, R.; Guinn, K. M.; Hickey, M. J.; Mohaideen, N.; Murphy, K. C.; Boshoff, H. I.; Mizrahi, V.; Rubin, E. J.; Sassetti, C. M.; Barry, C. E., 3rd; Sherman, D. R.; Parish, T.; Sacchettini, J. C. PLoS One 2013, 8, e75245.

[49] Aggarwal, A.; Parai, M. K.; Shetty, N.; Wallis, D.; Woolhiser, L.; Hastings, C.; Dutta, N. K.; Galaviz, S.; Dhakal, R. C.; Shrestha, R.; Wakabayashi, S.; Walpole, C.; Matthews, D.; Floyd, D.; Scullion, P.; Riley, J.; Epemolu, O.; Norval, S.; Snavely, T.; Robertson, G. T.; Rubin, E. J.; Ioerger, T. R.; Sirgel, F. A.; van der Merwe, R.; van Helden, P. D.; Keller, P.; Bottger, E. C.; Karakousis, P. C.; Lenaerts, A. J.; Sacchettini, J. C. Cell 2017, 170, 249.

[50] Zhang, W.; Lun, S.; Wang, S. H.; Jiang, X. W.; Yang, F.; Tang, J.; Manson, A. L.; Earl, A. M.; Gunosewoyo, H.; Bishai, W. R.; Yu, L. F. J. Med. Chem. 2018, 61, 791.

[51] Zhang, W.; Lun, S.; Liu, L. L.; Xiao, S.; Duan, G.; Gunosewoyo, H.; Yang, F.; Tang, J.; Bishai, W. R.; Yu, L. F. J. Med. Chem. 2019, 62,3575 .

[52] Dal Molin, M.; Selchow, P.; Schafle, D.; Tschumi, A.; Ryckmans, T.; Laage-Witt, S.; Sander, P. J. Mol. Med. (Berl.) 2019, 97, 1601.

[53] Nikaido, H. Res. Microbiol. 2018, 169, 363.

[54] Domenech, P.; Reed, M. B.; Barry, C. E. Infect. Immun. 2005, 73, 3492.

[55] Tullius, M. V.; Harmston, C. A.; Owens, C. P.; Chim, N.; Morse, R. P.; McMath, L. M.; Iniguez, A.; Kimmey, J. M.; Sawaya, M. R.; Whitelegge, J. P.; Horwitz, M. A.; Goulding, C. W. Proc. Natl. Acad. Sci. U. S. A. 2011, 108, 5051

[56] Degiacomi, G.; Benjak, A.; Madacki, J.; Boldrin, F.; Provvedi, R.; Palu, G.; Kordulakova, J.; Cole, S. T.; Manganelli, R. Sci. Rep. 2017, 7, 43495 .

[57] Grzegorzewicz, A. E.; Pham, H.; Gundi, V. A.; Scherman, M. S.; North, E. J.; Hess, T.; Jones, V.; Gruppo, V.; Born, S. E.; Kordulakova, J.; Chavadi, S. S.; Morisseau, C.; Lenaerts, A. J.; Lee, R. E.; McNeil, M. R.; Jackson, M. Nat. Chem. Biol. 2012, 8, 334.

[58] La Rosa, V.; Poce, G.; Canseco, J. O.; Buroni, S.; Pasca, M. R.; Biava, M.; Raju, R. M.; Porretta, G. C.; Alfonso, S.; Battilocchio, C.; Javid, B.; Sorrentino, F.; Ioerger, T. R.; Sacchettini, J. C.; Manetti, F.; Botta, M.; De Logu, A.; Rubin, E. J.; De Rossi, E. An- 
timicrob. Agents Chemother. 2012, 56, 324.

[59] Stanley, S. A.; Grant, S. S.; Kawate, T.; Iwase, N.; Shimizu, M.; Wivagg, C.; Silvis, M.; Kazyanskaya, E.; Aquadro, J.; Golas, A.; Fitzgerald, M.; Dai, H.; Zhang, L.; Hung, D. T. ACS Chem. Biol. 2012, 7, 1377.

[60] Tahlan, K.; Wilson, R.; Kastrinsky, D. B.; Arora, K.; Nair, V.; Fischer, E.; Barnes, S. W.; Walker, J. R.; Alland, D.; Barry, C. E., 3rd; Boshoff, H. I. Antimicrob. Agents Chemother. 2012, 56, 1797.

[61] Lun, S.; Guo, H.; Onajole, O. K.; Pieroni, M.; Gunosewoyo, H.; Chen, G.; Tipparaju, S. K.; Ammerman, N. C.; Kozikowski, A. P.; Bishai, W. R. Nat. Commun. 2013, 4, 2907.

[62] Rao, S. P. S.; Lakshminarayana, S. B.; Kondreddi, R. R.; Herve, M.; Camacho, L. R.; Bifani, P.; Kalapala, S. K.; Jiricek, J.; Ma, N. L.; Tan, B. H.; Ng, S. H.; Nanjundappa, M.; Ravindran, S.; Seah, P. G.; Thayalan, P.; Lim, S. H.; Lee, B. H.; Goh, A.; Barnes, W. S.; Chen, Z.; Gagaring, K.; Chatterjee, A. K.; Pethe, K.; Kuhen, K.; Walker, J.; Feng, G.; Babu, S.; Zhang, L. J.; Blasco, F.; Beer, D.; Weaver, M.; Dartois, V.; Glynne, R.; Dick, T.; Smith, P. W.; Diagana, T. T.; Manjunatha, U. H. Sci. Transl. Med. 2013, 5. 214.

[63] Remuinan, M. J.; Perez-Herran, E.; Rullas, J.; Alemparte, C.; Martinez-Hoyos, M.; Dow, D. J.; Afari, J.; Mehta, N.; Esquivias, J.; Jimenez, E.; Ortega-Muro, F.; Fraile-Gabaldon, M. T.; Spivey, V. L.; Loman, N. J.; Pallen, M. J.; Constantinidou, C.; Minick, D. J.; Cacho, M.; Rebollo-Lopez, M. J.; Gonzalez, C.; Sousa, V.; Angulo-Barturen, I.; Mendoza-Losana, A.; Barros, D.; Besra, G. S.; Ballell, L.; Cammack, N. PLoS One 2013, 8, e60933.

[64] Foss, M. H.; Pou, S.; Davidson, P. M.; Dunaj, J. L.; Winter, R. W.; Pou, S.; Licon, M. H.; Doh, J. K.; Li, Y.; Kelly, J. X.; Dodean, R. A.; Koop, D. R.; Riscoe, M. K.; Purdy, G. E. ACS Infect. Dis. 2016, 2, 500 .

[65] Dupont, C.; Viljoen, A.; Dubar, F.; Blaise, M.; Bernut, A.; Pawlik, A.; Bouchier, C.; Brosch, R.; Guerardel, Y.; Lelievre, J.; Ballell, L.; Herrmann, J. L.; Biot, C.; Kremer, L. Mol. Microbiol. 2016, 101, 515.

[66] Shetty, A.; Xu, Z.; Lakshmanan, U.; Hill, J.; Choong, M. L.; Chng, S. S.; Yamada, Y.; Poulsen, A.; Dick, T.; Gengenbacher, M. Front. Microbiol. 2018, 9, 2960.

[67] Zheng, H.; Williams, J. T.; Coulson, G. B.; Haiderer, E. R.; Abramovitch, R. B. Antimicrob. Agents Chemother. 2018, 62, 02459-17.

[68] Sacksteder, K. A.; Protopopova, M.; Barry, C. E.; Andries, K.; Nacy, C. A. Future Microbiol. 2012, 7, 823.

[69] Jia, J.; Tomaszewski, J. E.; Hanrahan, C.; Coward, L.; Noker, P.; Gorman, G.; Nikonenko, B.; Protopopova, M. Br. J. Pharmacol. 2005, 144, 80 .

[70] Reddy, V. M.; Einck, L.; Andries, K.; Nacy, C. A. Antimicrob. Agents Chemother. 2010, 54, 2840.

[71] Ballell, L.; Bates, R. H.; Young, R. J.; Alvarez-Gomez, D.; Alvarez-Ruiz, E.; Barroso, V.; Blanco, D.; Crespo, B.; Escribano, J.; Gonzalez, R.; Lozano, S.; Huss, S.; Santos-Villarejo, A.; Martin-Plaza, J. J.; Mendoza, A.; Rebollo-Lopez, M. J.; Remuinan-Blanco, M.; Lavandera, J. L.; Perez-Herran, E.; Gamo-Benito, F. J.; Garcia-Bustos, J. F.; Barros, D.; Castro, J. P.; Cammack, N. ChemMedChem 2013, 8, 313.

[72] Onajole, O. K.; Pieroni, M.; Tipparaju, S. K.; Lun, S.; Stec, J.; Chen, G.; Gunosewoyo, H.; Guo, H.; Ammerman, N. C.; Bishai, W. R.; Kozikowski, A. P. J. Med. Chem. 2013, 56, 4093.

[73] Kondreddi, R. R.; Jiricek, J.; Rao, S. P.; Lakshminarayana, S. B.;
Camacho, L. R.; Rao, R.; Herve, M.; Bifani, P.; Ma, N. L.; Kuhen, K.; Goh, A.; Chatterjee, A. K.; Dick, T.; Diagana, T. T.; Manjunatha, U. H.; Smith, P. W. J. Med. Chem. 2013, 56, 8849.

[74] Stec, J.; Onajole, O. K.; Lun, S.; Guo, H.; Merenbloom, B.; Vistoli, G.; Bishai, W. R.; Kozikowski, A. P. J. Med. Chem. 2016, 59, 6232.

[75] Li, W.; Sanchez-Hidalgo, A.; Jones, V.; de Moura, V. C.; North, E. J.; Jackson, M. Antimicrob. Agents Chemother. 2017, 61.

[76] Poce, G.; Cocozza, M.; Alfonso, S.; Consalvi, S.; Venditti, G.; Fernandez-Menendez, R.; Bates, R. H.; Barros Aguirre, D.; Ballell, L.; De Logu, A.; Vistoli, G.; Biava, M. Eur. J. Med. Chem. 2018, 145, 539.

[77] Scherman, M. S.; North, E. J.; Jones, V.; Hess, T. N.; Grzegorzewicz, A. E.; Kasagami, T.; Kim, I. H.; Merzlikin, O.; Lenaerts, A. J.; Lee, R. E.; Jackson, M.; Morisseau, C.; McNeil, M. R. Bioorg. Med. Chem. 2012, 20, 3255.

[78] Ramesh, R.; Shingare, R. D.; Kumar, V.; Anand, A.; B, S.; Veeraraghavan, S.; Viswanadha, S.; Ummanni, R.; Gokhale, R.; Srinivasa Reddy, D. Eur. J. Med. Chem. 2016, 122, 723.

[79] Zhang, B.; Li, J.; Yang, X.; Wu, L.; Zhang, J.; Yang, Y.; Zhao, Y.; Zhang, L.; Yang, X.; Yang, X.; Cheng, X.; Liu, Z.; Jiang, B.; Jiang, H.; Guddat, L. W.; Yang, H.; Rao, Z. Cell 2019, 176, 636.

[80] Li, W.; Stevens, C. M.; Pandya, A. N.; Darzynkiewicz, Z.; Bhattarai, P.; Tong, W.; Gonzalez-Juarrero, M.; North, E. J.; Zgurskaya, H. I.; Jackson, M. ACS Infect. Dis. 2019, 5, 1001.

[81] Ronning, D. R.; Vissa, V.; Besra, G. S.; Belisle, J. T.; Sacchettini, J. C. J. Biol. Chem. 2004, 279, 36771

[82] Anderson, D. H.; Harth, G.; Horwitz, M. A.; Eisenberg, D. J. Mol. Biol. 2001, 307, 671 .

[83] Content, J.; de la Cuvellerie, A.; De Wit, L.; Vincent-Levy-Frebault, V.; Ooms, J.; De Bruyn, J. Infect. Immun. 1991, 59, 3205.

[84] Jackson, M.; Raynaud, C.; Laneelle, M. A.; Guilhot, C.; Laurent-Winter, C.; Ensergueix, D.; Gicquel, B.; Daffe, M. Mol. Microbiol. 1999, 31, 1573.

[85] Warrier, T.; Tropis, M.; Werngren, J.; Diehl, A.; Gengenbacher, M.; Schlegel, B.; Schade, M.; Oschkinat, H.; Daffe, M.; Hoffner, S.; Eddine, A. N.; Kaufmann, S. H. Antimicrob. Agents Chemother. 2012, 56, 1735.

[86] Lehmann, J.; Cheng, T. Y.; Aggarwal, A.; Park, A. S.; Zeiler, E.; Raju, R. M.; Akopian, T.; Kandror, O.; Sacchettini, J. C.; Moody, D. B.; Rubin, E. J.; Sieber, S. A. Angew. Chem. Int. Ed. 2018, 57, 348.

[87] Liu, Y. G.; Matsumoto, M.; Ishida, H.; Ohguro, K.; Yoshitake, M.; Gupta, R.; Geiter, L.; Hafkin, J. Tuberculosis 2018, 111, 20.

[88] Ashtekar, D. R.; Costa-Perira, R.; Nagrajan, K.; Vishvanathan, N.; Bhatt, A. D.; Rittel, W. Antimicrob. Agents Chemother. 1993, 37, 183.

[89] Matsumoto, M.; Hashizume, H.; Tomishige, T.; Kawasaki, M.; Tsubouchi, H.; Sasaki, H.; Shimokawa, Y.; Komatsu, M. PLoS Med. 2006, 3, e466.

[90] Sasaki, H.; Haraguchi, Y.; Itotani, M.; Kuroda, H.; Hashizume, H.; Tomishige, T.; Kawasaki, M.; Matsumoto, M.; Komatsu, M.; Tsubouchi, H. J. Med. Chem. 2006, 49, 7854.

[91] Zhao, W.; Wang, B.; Liu, Y.; Fu, L.; Sheng, L.; Zhao, H.; Lu, Y.; Zhang, D. Eur. J. Med. Chem. 2020, 189, 112075.

[92] Conradie, F.; Diacon, A. H.; Ngubane, N.; Howell, P.; Everitt, D.; Crook, A. M.; Mendel, C. M.; Egizi, E.; Moreira, J.; Timm, J.; McHugh, T. D.; Wills, G. H.; Bateson, A.; Hunt, R.; Van Niekerk, C.; Li, M.; Olugbosi, M.; Spigelman, M.; Nix, T. B. T. T. N. Engl. J. Med. 2020, 382, 893 . 